\title{
The Classification of Nonsimple Algebraic Tangles
}

\author{
Ying-Qing $\mathrm{Wu}^{1}$
}

A tangle is a pair $(B, T)$, where $B$ is a 3 -ball, $T$ is a pair of properly embedded arcs. When there is no ambiguity we will simply say that $T$ is a tangle. Let $E(T)=B-$ $\operatorname{Int} N(T)$ be the exterior of $T$, usually called the tangle space. $T$ is simple if $E(T)$ is a simple manifold, that is, it is irreducible, $\partial$-irreducible, atoroidal, and anannular. By Thurston's geometrization theorem, simple tangle spaces admit hyperbolic structures with totally geodesic boundary. When embedding the tangle space into $S^{3}$ in the natural way, the complement is a handlebody of genus two. Hence, detecting simple tangles is the same as detecting embedded genus two handlebodies with simple complement.

In general, it is difficult to determine if a tangle is simple. The first simple tangle (see Figure 1.5(a)) was suggested by Jaco [7, P194], and was verified by Myers [8] with a rather lengthy argument. Due to the nature of the problem, such kind of argument seems unavoidable before a general theory of detecting simple tangles is developed. A similar tangle is the one in Figure 1.5(b), which was proved simple by Ruberman [10]. Another simple tangle is the one in Figure 1.6. Its tangle space was called the tripos manifold, and was proved to be hyperbolic by Thurston [12, Chapter 3]. These have been used by Adams and Reid [1] to discuss quasi-Fuchsian surfaces in knot complements. It seems that these are essentially the only tangles which were known to be simple.

A tangle is called an algebraic tangle if it can be obtained by summing up finitely many rational tangles in various ways. An algebraic tangle is a Montesinos tangle if all the gluing disks are disjoint from each other. By analyzing incompressible annuli in exteriors of algebraic tangles, we will be able to prove Theorem 4.9, which completely classifies all nonsimple algebraic tangles.

The paper is organized as follows. In Section 1 we will state the classification theorems, prove corollaries, and show some examples. Section 2 is to classify all marked tangles containing either a monogon or a bigon. The results will be used in Section 3 to give the proofs of Theorem 3.6. In Section 4 we will determine all marked algebraic tangles which are $\Delta$-annular (see Section 1 for definitions), and use the result to prove the classification theorem of nonsimple algebraic tangles.

\footnotetext{
${ }^{0}$ Mathematics Subject Classification: 57N10, 57M25, 57M50.

${ }^{1}$ Partially supported by NSF grant DMS 9102633
} 
I would like to thank John Luecke for some helpful discussions on the topic.

\section{Main theorems and examples}

Let $(B, T)$ be a tangle. Fix an embedding of $B$ in $R^{3}$, and fix 4 points $\mathcal{P}=\{a, b, c, d\}$ as shown in Figure 1.1. Two tangles $(B, T)$ and $\left(B^{\prime}, T^{\prime}\right)$ are said to be equivalent or $w$ equivalent if there is a homeomorphism $\varphi:(B, T) \rightarrow\left(B^{\prime}, T^{\prime}\right)$. They are s-equivalent if $\left.\varphi\right|_{\partial B}$ is the identity map.

The double cover of $\partial B$ branched over $\mathcal{P}$ is a torus, whose universal cover is $\mathbf{R}^{2}$. This gives rise to a map $\varphi: \mathbf{R}^{2} \rightarrow \partial B$ such that $\varphi^{-1}(\mathcal{P})$ is the set of integral points. If $l$ is a line with slope a rational number $r$, then $\varphi(l)$ is a circle or an arc connecting two points of $\mathcal{P}$. We say that $\varphi(l)$ has slope $r$. Thus the curve $C_{x}$ and $C_{y}$ in Figure 1.1 have slopes 0 and $\infty$ respectively.

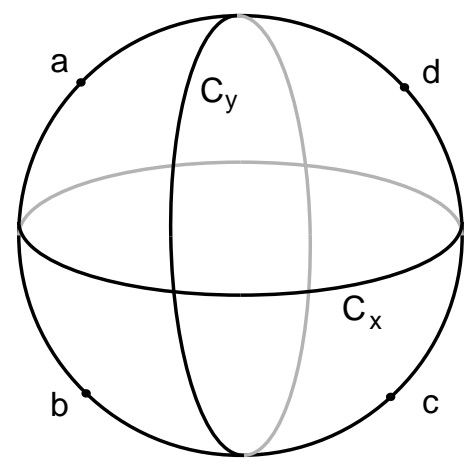

Figure 1.1

$(B, T)$ is a rational tangle of slope $r$ if $T$ is rel $\partial T$ isotopic to two slope $r \operatorname{arcs}$ on $\partial B$. In this case we denote it by $M(r)$. Since all rational tangles are w-equivalent to the "trivial" one in Figure 1.2(a), they will also be called trivial tangles.

A marked tangle is a triple $(B, T, \Delta)$, where $(B, T)$ is a tangle, and $\Delta$ is a disk on $\partial B$ containing two endpoints of $T . \Delta$ is called the gluing disk. Two marked tangles are equivalent if there is a homeomorphism of triples. Given a tangle $(B, T)$, the two disks on $\partial B$ on the left and right of $C_{y}$ are called the left disk and right disk, respectively. Unless otherwise stated, we will always choose the left disk as a gluing disk. If $M(r)$ is a rational tangle, then we use $M[r]$ to denote the corresponding marked rational tangle. By definition it is clear that $M[r]=M\left[r^{\prime}\right]$ if and only if $r \equiv r^{\prime} \bmod \mathbf{Z}$. Thus the tangles in Figure 1.2 are $M[0], M[\infty]$ and $M[1 / 5]$ respectively. The tangle $M[1 / q]$ is called a $q$-twist tangle. 
Given two marked tangles $\left(B_{1}, T_{1}, \Delta_{1}\right)$ and $\left(B_{2}, T_{2}, \Delta_{2}\right)$, we may choose a map $\varphi: \Delta_{1} \rightarrow$ $\Delta_{2}$ with $\varphi\left(\Delta_{1} \cap T_{1}\right)=\Delta_{2} \cap T_{2}$, and use it to glue the two tangles together to get a new tangle $(B, T)$. We say that $(B, T)$ is the sum of $\left(B_{1}, T_{1}, \Delta_{1}\right)$ and $\left(B_{2}, T_{2}, \Delta_{2}\right)$, or simply that $T$ is the sum of $T_{1}$ and $T_{2}$, and write $(B, T)=\left(B_{1}, T_{1}, \Delta_{1}\right)+\left(B_{2}, T_{2}, \Delta_{2}\right)$, or $T=T_{1}+T_{2}$. Note that the sum $T=T_{1}+T_{2}$ depends on the choice of the gluing disks $\Delta_{i}$ on $\partial B_{i}$, and the gluing map $\varphi$. It is called a nontrivial sum if neither $\left(B_{i}, T_{i}, \Delta_{i}\right)$ is $M[0]$ or $M[\infty]$.

Given rational tangles $M\left(r_{1}\right) \ldots, M\left(r_{k}\right)$, we can glue the right disk of $M\left(r_{i}\right)$ to the left disk of $M\left(r_{i+1}\right)$ to get a tangle $M\left(r_{1}, \ldots, r_{k}\right)$, called a Montesinos tangle. To avoid trivial sums, we will always assume that $r_{i}$ are non-integral rational numbers.
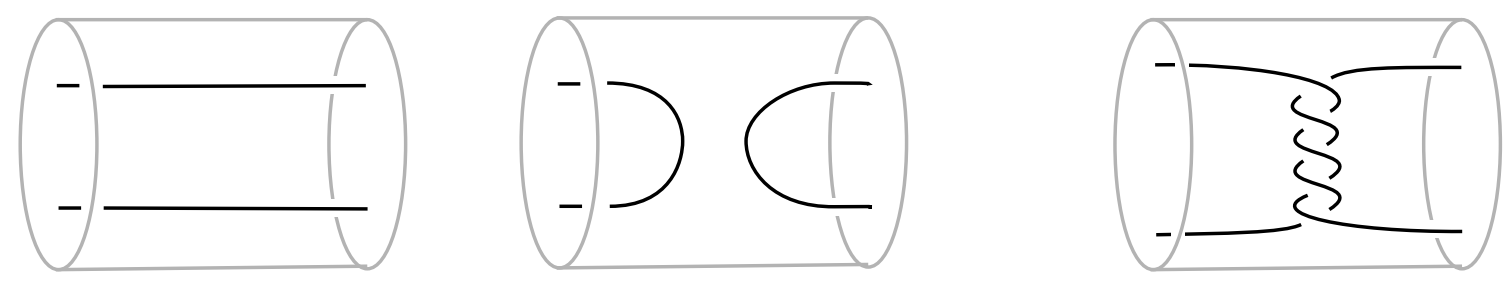

Figure 1.2

We will use $E(T)$ to denote the exterior of $T$, i.e. $E(T)=B-\operatorname{Int} N(T)$. Similarly, if $T=t_{1} \cup t_{2}$, then $E\left(t_{i}\right)=B-\operatorname{Int} N\left(t_{i}\right)$.

There is a smallest class of tangles which contains all the rational tangles, and is closed under nontrivial tangle sums. A tangle in this class is called an algebraic tangle. In other words, a tangle is algebraic if it is a sum of finitely many rational tangles. An algebraic tangle is a Montesinos tangle if and only if all the gluing disks are disjoint to each other.

We define the length of an algebraic tangle $T$ to be the least number $L(T)$ so that $T$ is a sum of $L(T)$ rational tangles. When $L(T)=1, T$ is a rational tangle.

Suppose that $r_{1}$ and $r_{2}$ are non-integral rational numbers. For $r_{3}=1 / q$ or 0 , we define a class of marked tangle $R\left[r_{1}, r_{2} ; r_{3}\right]$ so that its underlying tangle is the Montesinos tangle $M\left(r_{1}, r_{2}\right)$, its gluing disk $\Delta$ contains the lower left end of the strings, and $\partial \Delta$ has slope $r_{3}$ on $\partial B$. One can see that as marked tangles they are equivalent to the tangles in Figure 1.3 , with left disk as gluing disk. The tangle on the left is $R\left[r_{1}, r_{2} ; r_{3}\right]$ with $r_{3}=1 / q$, and the one on the right is $R\left[r_{1}, r_{2} ; 0\right]$. 

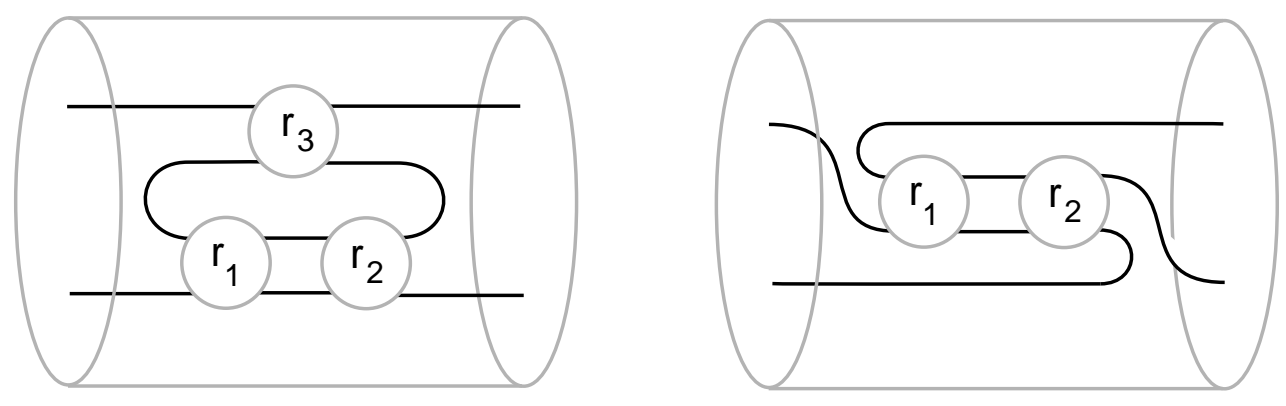

Figure 1.3

Given numbers $r_{1}, r_{2}, r_{3}$, the four tangles $R\left[r_{1}, r_{2} ; r_{3}\right], R\left[-r_{1},-r_{2} ;-r_{3}\right], R\left[r_{2}, r_{1} ; r_{3}\right]$ and $R\left[-r_{2},-r_{1} ;-r_{3}\right]$ are all said to be similar to $R\left[r_{1}, r_{2} ; r_{3}\right]$. One can see that $R\left[-r_{1},-r_{2} ;-r_{3}\right]$ is a mirror image of $R\left[r_{1}, r_{2} ; r_{3}\right]$, and $R\left[r_{2}, r_{1} ; r_{3}\right]$ can be obtained from $R\left[r_{1}, r_{2} ; r_{3}\right]$ by taking the right disk as the gluing disk. The tangle $M[r]$ is similar to itself.

Let $\mathcal{S}$ be the set of those tangles which are similar to one of the following.

(1) $R\left[\frac{1}{2}, \frac{1}{q} ;-\frac{1}{2}\right]$, where $|q| \geq 3$;

(2) $R\left[\frac{1}{2},-\frac{1}{3} ;-\frac{1}{4}\right]$;

(3) $R\left[\frac{1}{2},-\frac{1}{3} ;-\frac{1}{6}\right]$;

(4) $R\left[\frac{2}{3},-\frac{1}{3} ;-\frac{1}{3}\right]$;

(5) $R\left[\frac{1}{3},-\frac{1}{3} ; 0\right]$;

(6) $M\left[\frac{1}{2 n}\right]$ with $|n| \geq 2$.

Notice that every tangle in $\mathcal{S}$ has the property that one of its strings has both ends on the gluing disk. In practice this is helpful to determine whether a tangle is in $\mathcal{S}$ (see Example 4).

We can now state the classification theorem of nonsimple algebraic tangles. Recall that a tangle is a trivial tangle if and only if it is a rational tangle.

Theorem 4.9. A nontrivial algebraic tangle $T$ is nonsimple if and only if one of the following holds.

(a) $T=M\left(\frac{1}{2}, \frac{p}{q}\right)$;

(b) $T=M\left(\frac{1}{q}, \frac{1}{q^{\prime}}\right)$, $q$ and $q^{\prime}$ are odd numbers;

(c) $T=T_{1}+T_{2}$, each $T_{i}$ is $R\left[\frac{1}{2},-\frac{1}{3} ; 0\right]$ or $R\left[-\frac{1}{2}, \frac{1}{3} ; 0\right]$, and the unknotted string of $T_{1}$ is glued to the unknotted string of $T_{2}$;

(d) $T=T_{1}+T_{2}$, and $T_{1} \in \mathcal{S}$.

Corollary 1.1 Let $M\left(r_{1}, \ldots r_{m}\right)$ be a Montesinos tangle with non-integral $r_{i}$. 
(a) If $m=2, M\left(r_{1}, r_{2}\right)$ is nonsimple if and only if either both $M\left[r_{i}\right]$ are twist tangles, or one of them is a (2n)-twist tangle, $n \neq 0$.

(b) If $m \geq 3, M\left(r_{1}, \ldots, r_{m}\right)$ is nonsimple if and only if either $M\left[r_{1}\right]$ or $M\left[r_{m}\right]$ is a (2n)-twist tangle with $|n| \geq 2$.

Proof. If $(B, T)=\left(B^{\prime}, T^{\prime}, \Delta^{\prime}\right)+\left(B^{\prime \prime}, T^{\prime \prime}, \Delta^{\prime \prime}\right)$ is a nontrivial sum, we call the disk $\Delta^{\prime}=\Delta^{\prime \prime}$ a cutting disk of $(B, T)$. Two disjoint cutting disks $\Delta_{1}, \Delta_{2}$ are called parallel if the part of $(B, T)$ between then is a product $\Delta_{1} \times I$. A set of cutting disks $\Delta_{1} \cup \ldots \cup \Delta_{k}$ is called a maximal cutting set if they are mutually disjoint, mutually nonparallel, and is maximal subject to these conditions. It is easy to show that if a tangle is atoroidal, then up to isotopy of the pair $(B, T)$ there is a unique maximal cutting set $\mathcal{C}$ (possibly empty). Thus up to isotopy any cutting disk is contained in $\mathcal{C}$. A Montesinos tangle is characterized by the fact that $\mathcal{C}$ cuts $(B, T)$ into rational tangles.

A nontrivial sum of two tangles can not be a rational tangle (see for example Lemma 3.3 below.) Hence (a) follows immediately from Theorem 4.9 .

Now suppose $T=M\left(r_{1}, \ldots, r_{m}\right)$ is nonsimple, $m \geq 3$, and suppose $M\left[r_{1}\right]$ and $M\left[r_{m}\right]$ are not (2n)-twist tangles with $|n| \geq 2$. Then by Theorem $4.9,(B, T)$ is a sum $\left(B_{1}, T_{1}, \Delta_{1}\right)+$ $\left(B_{2}, T_{2}, \Delta_{2}\right)$, and $T_{1}$ is of the type $R\left[r_{1}, r_{2} ; r_{3}\right]$. Since $r_{3} \neq \infty$, the gluing disk of $R\left[r_{1}, r_{2} ; r_{3}\right]$ has to intersect the (unique) cutting disk in $M\left(r_{1}, r_{2}\right)$. Thus if $\mathcal{C}$ is a maximal cutting set of $(B, T)$ containing $\Delta_{1}$, then $\mathcal{C} \cap B_{1}=\Delta_{1}$, so $\left(B_{1}, T_{1}\right)=M\left(r_{1}, r_{2}\right)$ is a component of $(B, T)$ cutting along $\mathcal{C}$. Since $M\left(r_{1}, r_{2}\right)$ is not a rational tangle, it follows that $(B, T)$ is not a Montesinos tangle.

Example 1. The tangle in Figure 1.4 is a sum of a 3 -twist tangle and a (-3)-twist tangle. It was suggested to be simple in [7, P194], but it was noticed by Adams and Reid that it contains an essential annulus. Their annulus is different from that shown in Figure 1.4.
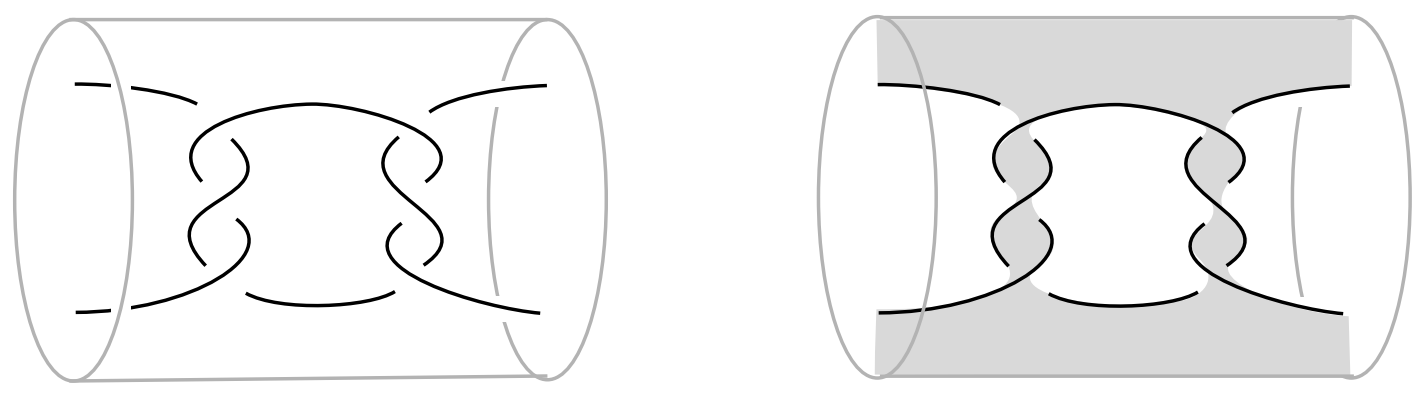

Figure 1.4 
Example 2. The tangle in Figure 1.5(a) was suggested to be simple in [7, Page 194], and was proved so by Myers [8]. It is Montesinos tangle of length 3, and the two end tangles are not even-twist tangles, so Corollary 1.1 tells immediately that it is simple. Similarly, the tangle in Figure 1.5(b) is simple, which was first proved by Ruberman [10].

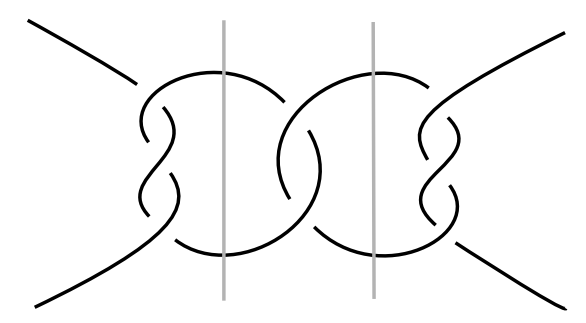

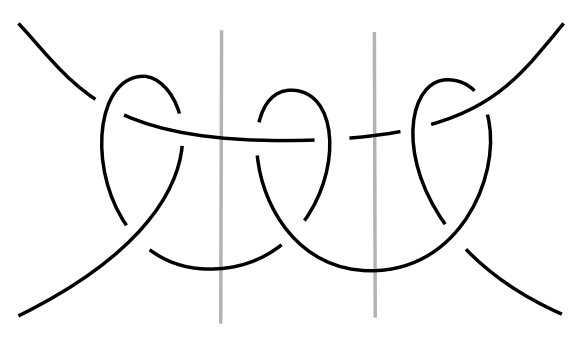

Figure 1.5

Example 3. The exterior of the graph in Figure 1.6(a) was shown by Thurston [12, Chapter $3]$ to admit a hyperbolic structure with totally geodesic boundary, so it is simple. This also follows from the above results. By shrinking one of the edges to a point then removing a neighborhood of it, one can see that the exterior of the graph is the same as the exterior of the tangle in Figure 1.6(b), which is a sum of two rational tangles. The associated rational numbers are $2 / 5$ and $1 / 3$, respectively, so by Corollary 1.1 the sum is a simple tangle.
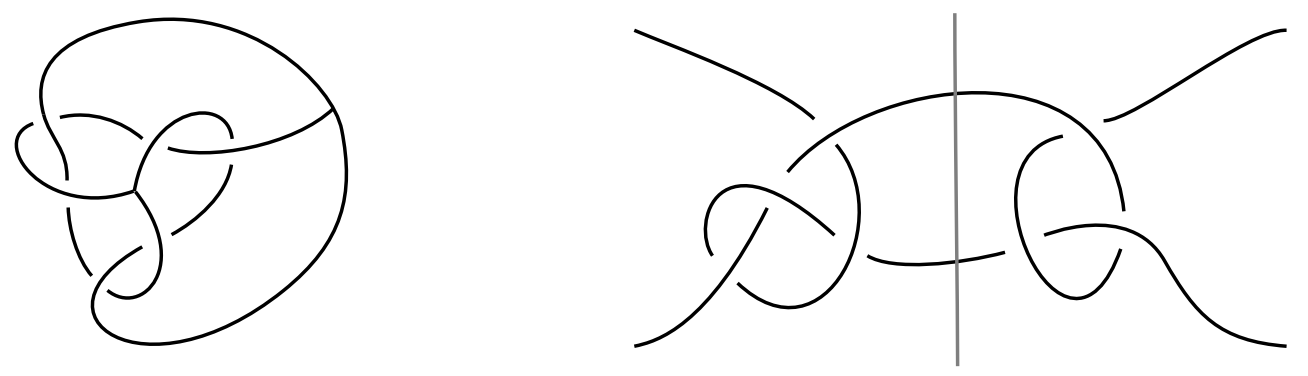

Figure 1.6

Example 4. The left hand side of the tangle in Figure 1.7(a) is a nontrivial sum of two tangles, so it is not a rational tangle. It can not be in $\mathcal{S}$ either because its two ends on the gluing disk are on different strings. By Theorem 4.9 the sum is simple. It can be converted to that in Figure 1.7(b). This is probably the "simplest" simple tangle as it has a projecting diagram with only six crossings. 

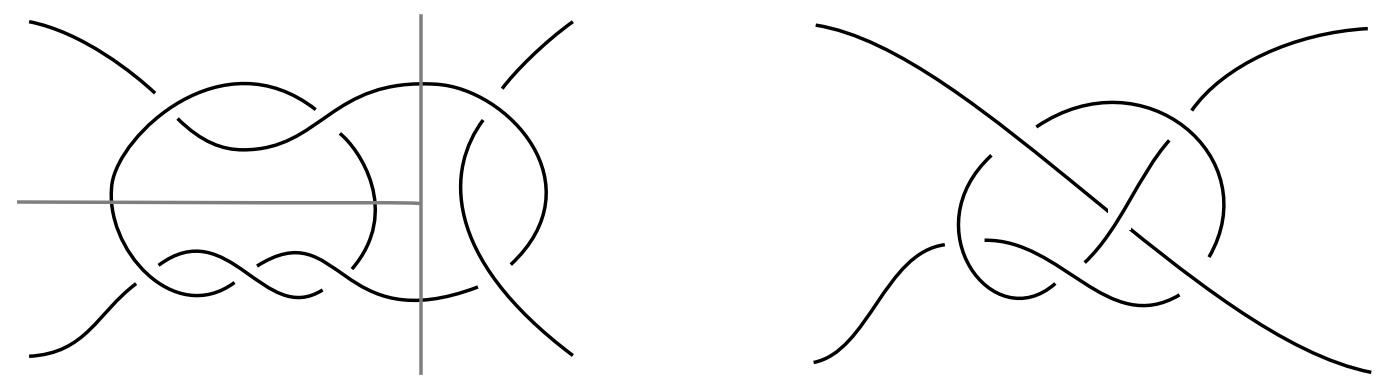

Figure 1.7

Suppose $T=T_{1}+T_{2}$ is a nontrivial sum. It is easy to show that if one of the $T_{i}$ has an essential torus, then it remains essential in $T$. Therefore a necessary condition for $T$ to be simple is that both $T_{i}$ are atoroidal. Theorem 3.6 classifies all nonsimple sums of such tangles. It will be applied to prove Theorem 4.9. We need the following definitions.

Definition. (1) A marked tangle $(B, T, \Delta)$ is called a wrapping tangle if (i) one of the string $t_{1}$ is unknotted and has exactly one end on $\Delta$; (ii) $t_{2}$ is rel $\partial t_{2}$ isotopic in $E\left(t_{1}\right)$ to an arc on $\partial E\left(t_{1}\right)$ which intersects each of $\Delta$ and $\partial N\left(t_{1}\right)$ in two arcs; and (iii) it is nontrivial. See Figure 1.8(a) for a typical example of the arc to which $t_{2}$ is isotopic. The tangle in Figure 1.8 (b) is equivalent to the tangle of 1.8(a). The wrapping number of $T$ is defined intuitively as the number of times the knotted string wrapping around the unknotted one. Thus the tangle in Figure 1.8(b) is a 3-wrapping tangle, and its mirror image is a (-3)-wrapping tangle. When the wrapping number is 1 , the picture can be simplified to that in Figure $1.8(\mathrm{c})$, which is the simplest nontrivial wrapping tangle. Notice that a 1-wrapping tangle is equivalent to $R\left[-\frac{1}{2}, \frac{1}{3} ; 0\right]$.
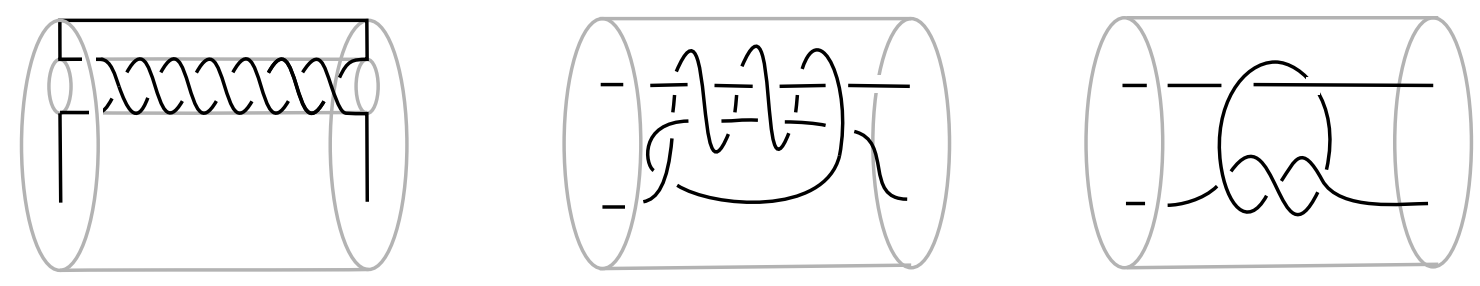

Figure 1.8

(2) $(B, T, \Delta)$ is called a torus tangle if (i) one of the string $t_{1}$ has both ends on either $\Delta$ or $\partial B-\Delta$, and is unknotted (i.e $E\left(t_{1}\right)$ is a solid torus); (ii) there is an $\operatorname{arc} \alpha$ on $\partial B-\partial \Delta$ 
connecting the two ends of $t_{2}$, and $t_{2}$ is rel $\partial t_{2}$ isotopic in $E\left(t_{1}\right)$ to an $\operatorname{arc} \beta$ on $\partial E\left(t_{1}\right)$ which meets $\alpha$ only at its endpoints. Thus $\alpha \cup \beta$ is a $(p, q)$ curve with respect to the standard longitude-meridian pair of the torus $\partial E\left(t_{1}\right)$. When $p$ or $q$ is \pm 1 , it is a twist tangle. To avoid trivial cases, we further assume (iii) $p \geq 2$, and $|q| \geq 2$. $T$ is called a $(p, q)$ torus tangle. There are actually two such tangles. When $l_{1}$ has ends on $\partial B-\Delta$ (resp. $\Delta$ ), it is called a left (resp. right) torus tangle. See Figure 1.9 for a $(2,5)$ left torus tangle.

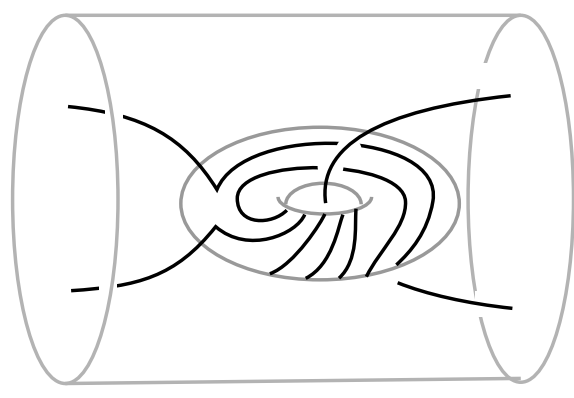

Figure 1.9

(3) Let $(B, T, \Delta)$ be a marked tangle. A properly embedded annulus $A$ in $E(T)$ is called $\Delta$-essential if (i) $A$ is incompressible, (ii) $\partial A$ is disjoint from the surface $P=\Delta \cap E(T)$, and (iii) there is no $\partial$-compressing disk of $A$ disjoint from $P$.

(4) $(B, T, \Delta)$ is $\Delta$-anannular if $E(T)$ contains no $\Delta$-essential annulus. Otherwise it is $\Delta$-annular.

Theorem 3.6. Suppose $T=T_{1}+T_{2}$ is a nontrivial sum of atoroidal tangles. Then $T$ is nonsimple if and only if, up to relabeling of $T_{i}$, one of the following holds.

(1) $T_{1}$ is a 2-twist tangle, and $T_{2}$ is a rational tangle;

(2) Both $T_{i}$ are twist tangles;

(3) Both $T_{i}$ are wrapping tangles, and the unknotted string of $T_{1}$ is glued to the unknotted string of $T_{2}$;

(4) $T_{1}$ is $\Delta$-annular.

Remark. In case (1) of the theorem, the tangle space is a handlebody. In case (2) and (3), the tangle space contains an essential annulus except when one of the $T_{i}$ in case (2) is a 2 -twist tangle, in which case it is a handlebody. When both $T_{i}$ are twist tangles, an annulus or Möbius band is shown in Fig 1.4. Notice that when $E(T)$ has a Möbius band, the frontier of its regular neighborhood is an annulus. Similarly, if $T_{1}$ and $T_{2}$ are both twist tangles or both wrapping tangles, then an annulus or Möbius band can be formed by putting two 
bigons together. See section 2 for details about bigons. We will show in Lemma 3.5 that these annuli are essential unless one of the $T_{i}$ is a 2 -twist tangle. In case (4), a $\Delta$-essential annulus of $T_{1}$ becomes an essential annulus in $E(T)$.

\section{Marked tangles containing monogons or bigons}

Suppose $(B, T, \Delta)$ is a marked tangle. We use $E=E(T)$ the denote the tangle space, and $E\left(t_{i}\right)$ the exterior of the string $t_{i}$. Let $A_{i}$ be the annulus $N\left(t_{i}\right) \cap E$, let $P$ be the twice punctured disk $\Delta \cap \partial E$, and $Q$ the closure of $\partial E-\left(P \cup A_{1} \cup A_{2}\right)$. Thus, $\partial E$ is decomposed into four parts: $A_{1}, A_{2}, P$ and $Q$. A properly embedded surface $F$ in $E$ is in general position if the intersection of $\partial F$ with each of the four surfaces consists of essential arcs and/or essential circles. If $F$ is a compressing disk or essential annulus in $E$, then it can be isotoped to be in general position, so we will always assume this below.

A compressing disk $D$ of $\partial E$ is called a monogon of $E$ if $\partial D$ intersects $P$ in a single arc; it is a bigon if $\partial D$ intersects $P$ in two arcs. We call $\partial_{0}=P \cap Q$ the outer boundary of $P$, and call the other two components $\partial_{1}, \partial_{2}$ of $\partial P$ the inner boundary. The situation is quite simple when $E$ has a monogon.

Lemma 2.1 If a nontrivial atoroidal tangle $T$ contains a monogon $D$, then it is a 2-twist tangle, and $\partial D \cap P$ is an arc with both ends on the outer boundary of $P$.

Proof. Let $\alpha$ be the arc $\partial D \cap P$. There are four possibilities:

(i) $\alpha$ has one end on each of $\partial_{1}$ and $\partial_{2}$;

(ii) $\alpha$ has both ends on $\partial_{1}$ or $\partial_{2}$;

(iii) $\alpha$ has exactly one end on $\partial_{0}$;

(iv) $\alpha$ has both ends on $\partial_{0}$.

One can check that in the first three cases, $T$ would be either $M[0]$ or $M[\infty]$. So we concentrate on Case (iv). We want to show that in this case $T$ is a 2 -twist tangle.

By our convention $D$ is in general position. In particular, both boundary components of $A_{i}$ intersects $\partial D$ at the same number of points. If $\partial D$ is disjoint from both $A_{i}$, one can see that $T$ is a trivial tangle, contradicting the assumption. Thus the two circles $\partial_{1}$ and $\partial_{2}$ on $P$ must be on the same annulus, say $A_{1}$, because they are both disjoint from $\partial D$. Consider the arcs $Q \cap \partial D$ on $Q$. Since none of the arcs is inessential, and since the two circles $\partial A_{2}$ contain the same number of endpoints of the arcs, it is easy to see that, except for the two arcs running from $\partial \alpha$ to $\partial A_{2}$, all the other arcs run from one component of $\partial A_{2}$ to another. Suppose there are $k$ such arcs. Then $\partial D^{\prime}$ intersects a meridian of $A_{2}$ at $k+1$ points in the same direction, so a neighborhood of $\partial B \cup N\left(t_{2}\right) \cup D$ would be a punctured lens space in $B$ 
with first homology $\mathbf{Z}_{k+1}$, which is absurd unless $k=0$. Therefore, $\partial D$ intersects $A_{2}$ just once. Such a disk can be used to isotop $t_{2}$ to the $\operatorname{arc} \partial D \cap \partial E$. Since $T$ is atoroidal, the other string is also unknotted. It is now easy to see that $T$ is a 2-twist tangle.

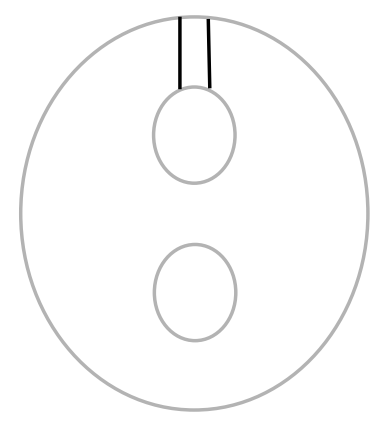

(1)

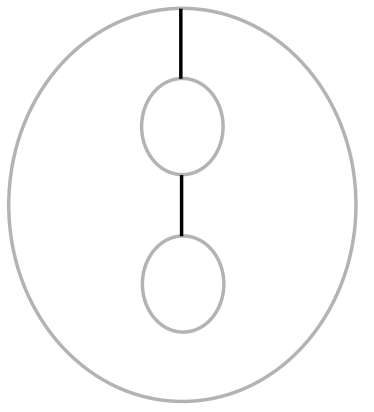

(4)

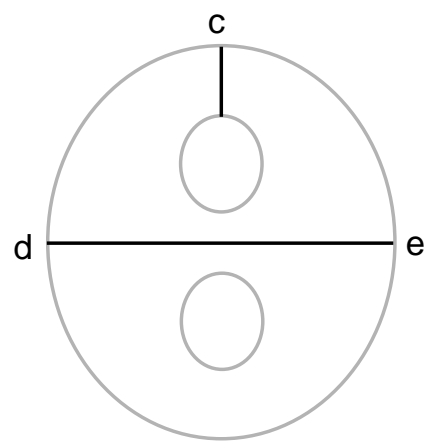

(7)

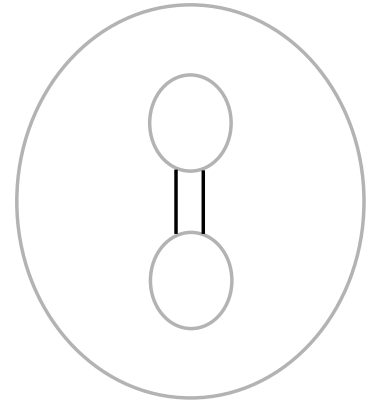

(2)

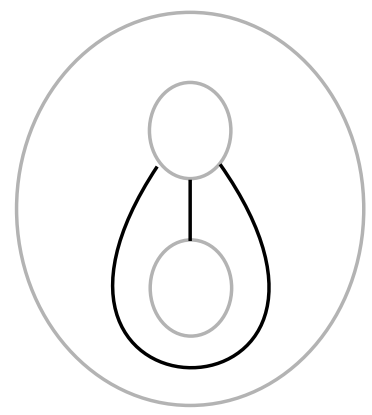

(5)

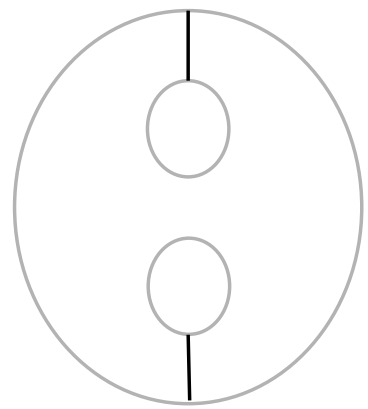

(8)

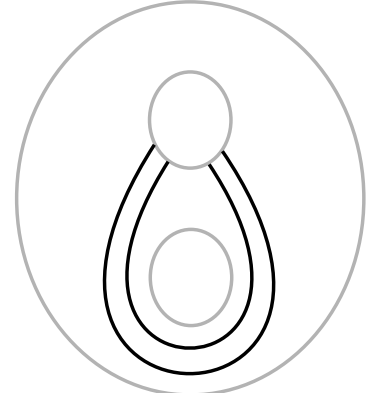

(3)

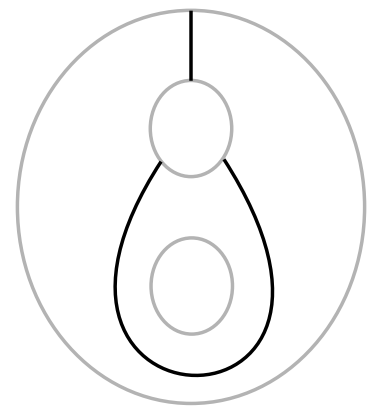

(6)

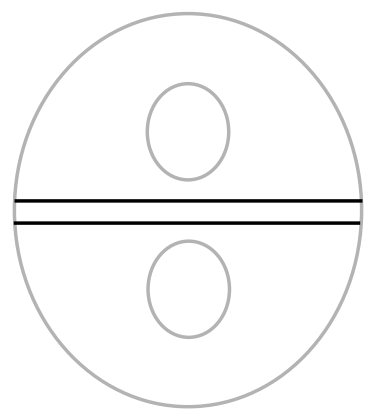

(9)

Figure 2.1

Now suppose $E$ contains a bigon $D$, with $\alpha_{1}, \alpha_{2}$ the two $\operatorname{arcs}$ of $\partial D \cap P$. Up to homeomorphism of $D$ there are nine possibilities for the configuration of $\alpha_{1}, \alpha_{2}$, as shown in Figure 
2.1 (1)-(9), where the outer circle on each figure represents the boundary component of $P$ which lies on $Q$. Label this boundary of $P$ as $\partial_{0}$, let $\partial_{1}$ be the upper inner boundary, and $\partial_{2}$ the lower inner boundary. The two inner boundaries may or may not be on the same annulus, but if they have different number of intersection points with $\partial D$, then they are definitely on different $A_{i}$ 's, in which case we assume $\partial_{i}$ lies on the boundary of $A_{i}$. The following lemma classifies all atoroidal tangles containing a bigon.

Lemma 2.2 Suppose $(B, T, \Delta)$ is a marked atoroidal tangle which contains a bigon. Cases (1)-(3) in Figure 2.1 cannot happen. In cases (4)-(6), $T$ is a wrapping tangle with $t_{1}$ the unknotted string. In case (7)-(9) it is either a twist tangle or a left torus tangle.

Proof. Notice that if one of the string, say $t_{2}$, can be isotoped in $E\left(t_{1}\right)$ to an arc on $\partial E\left(t_{1}\right)$, then $E\left(t_{1}\right)$ can be embedded into $E$. Since $E$ is irreducible and atoroidal, the image of $\partial E\left(t_{1}\right)$ bounds a solid torus, so $E\left(t_{1}\right)$ must be a solid torus, that is $t_{1}$ is a trivial arc in $B$. We discuss the nine cases separately.

Case (1). In this case, $\partial D$ is disjoint from $A_{2}$, and intersect $A_{1}$ twice. Since the two arcs of $\partial D$ on $Q$ are essential, one can check that each of them has ends on different components of $\partial Q$, so $\partial D$ intersects $A_{1}$ twice in the same direction. A neighborhood of $D \cup N\left(t_{1}\right) \cup \partial B$ would then be a punctured lens space in the 3-ball $B$, which is absurd.

Case (2). If the two circles in $P$ are the boundary of a single annulus $A_{i}$, one can get contradiction just as in Case (1). So assume they are on different $A_{i}$. As before one can show that $\partial D$ intersects each $A_{i}$ twice in the same direction. In particular, $D$ is a nonseparating disk in $E$. The set $E-\operatorname{Int} N(D)$ is contained in $E$ and bounded by a torus, so by atoroidality of $E$ it is a solid torus. Let $D^{\prime}$ be another disk so that $D$ and $D^{\prime}$ cut $E$ into a 3 -cell. Then $B$ can be obtained from $(\partial B) \cup N(T)$ by adding the two 2-handles $D$ and $D^{\prime}$, then attaching a 3 -cell. Each $t_{i}$ represents a generator of the first homology of $(\partial B) \cup N(T)$, and $\partial D$ represents $2 t_{1}+2 t_{2}$. Thus the homology after adding both $D$ and $D^{\prime}$ would either be infinite or have a $\mathbf{Z}_{2}$ subgroup. But since the 3 -ball $B$ is obtained by attaching a 3-cell onto this space, the group should be trivial, a contradiction.

Case (3). Consider the two essential $\operatorname{arcs} \beta_{1}, \beta_{2}$ of $\partial D$ on $Q$. One can see that they lie on $Q$ in the same pattern as $\alpha_{1}, \alpha_{2}$ lie on $P$. The four arcs of $\partial D$ on $A_{1}$ connect the ends of $\alpha_{i}$ to that of $\beta_{i}$ with a permutation so that the union is a connected curve. One can check that when considering $\partial D$ as a curve on the torus $\left.\partial E\left(t_{1}\right)\right)$, it is isotopic to a meridian of $A_{1}$. But such a curve can never bound a disk in $B-\operatorname{Int} N\left(t_{1}\right)$, so it could not have bound a disk in $E$.

Case (4). As the two circles in $P$ intersect $\partial D$ in different number of points, they belong to different annuli $A_{i}$. Recall our convention that in this case $A_{2}$ is the annulus which 
contains $\partial_{2}$, the lower inner circle, as a boundary component. Since $D$ intersects $A_{2}$ in a single essential arc, the string $t_{2}$ can be isotoped through $D$ to the $\operatorname{arc} \alpha=\partial D-A_{2}$ on the boundary of $E\left(t_{1}\right)$. As we noticed at the beginning of the proof, this implies that the arc $t_{1}$ is an unknotted arc. Since $\alpha$ intersects each of $D$ and $A_{1}$ twice, $T$ is a wrapping tangle by definition.

Case (5). Suppose $A_{2}$ is the annulus intersecting $\partial D$ at a single arc. The string $t_{2}$ can be isotoped through $D$ to lie on the boundary of $E\left(t_{1}\right)$, so by the remark at the beginning of the proof, $t_{1}$ is unknotted. Up to homeomorphism of $B$ fixing $\Delta$ there are two choices for the $\operatorname{arcs} \partial D \cap A_{1}$ on $A_{1}$. One is that shown in Figure 2.2, the other is its mirror image. Since $t_{2}$ is isotopic to $\partial D \cap \partial E\left(t_{1}\right)$, it is isotopic to the knotted arc in Figure 1.8(c). Therefore, $T$ is a 1-wrapping tangle.

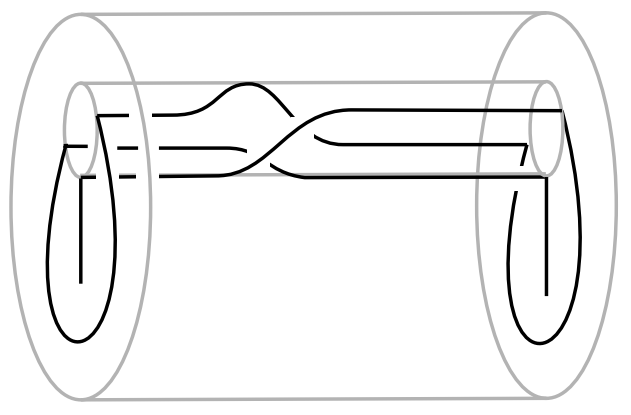

Figure 2.2

Case (6). Since $\partial D$ intersects $\partial_{0}$ at one point, it is a nonseparating disk. So when cutting $E\left(t_{1}\right)$ along the disk $D$, we get a manifold in $B$ bounded by a sphere, which must be a 3 -ball $B^{\prime}$. Since $T$ is atoroidal, the arc $t_{2}$ is unknotted in $B^{\prime}$. Hence there is a disk $D^{\prime}$ in $B^{\prime}$ such that $\partial D^{\prime}=t_{2} \cup \beta$, where $\beta$ can be any arc on $\partial B^{\prime}$, so it can be any arc on $\partial E\left(t_{1}\right)$ which is disjoint from $\partial D$ and connects the two ends of $t_{2}$. One can draw such a curve $\beta$ so that $\beta \cap P$ consists of two arcs as that in Figure 2.1(5). Therefore $D^{\prime}$ gives rise to a bigon of Case (5), and $T$ is a 1-wrapping tangle, as we have just shown.

Case (7). Again the two circles belong to different annuli, and $t_{1}$ can be isotoped to an $\operatorname{arc} \alpha=\partial D \cap \partial B$ on the boundary of $E\left(t_{2}\right)=B-\operatorname{Int} N\left(t_{2}\right)$. The intersection $\alpha \cap Q$ consists of two arcs, one arc $b_{1}$ connects the point $c$ in Figure 2.1(7) to either $d$ or $e$, and the other one $b_{2}$ connects $d$ or $e$ to the end of $t_{1}$ on $Q$. We assume the later. By a homeomorphism of $(B, \Delta)$ fixing $\Delta$ and twisting on $\partial B-\Delta$, we may assume that $b_{2}$ looks like that in Figure 
2.3. Now there is only one way to draw $b_{1}$, as in the figure. Since the tangle is atoroidal, the string $t_{2}$ is unknotted, so the tangle space looks exactly like that in the figure, with the boundary arc replaced by a nearby string. One can see that $T$ is a 3 -twist tangle. Similarly, if the arc $b_{1}$ connects $c$ to $d, T$ is a (-3)-twist tangle.

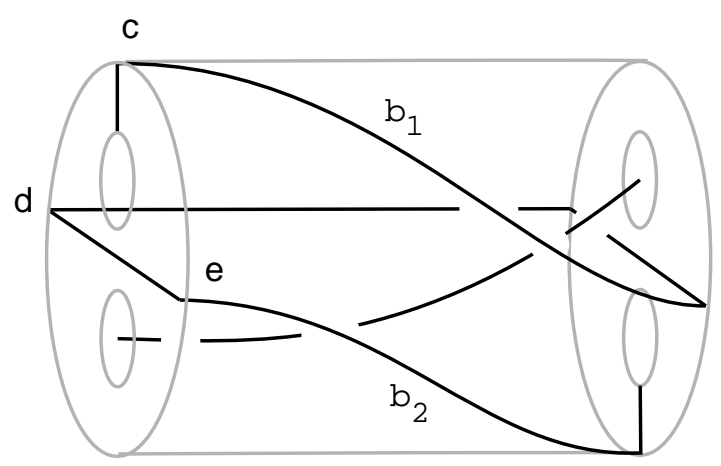

Figure 2.3

Case (8). There are two possibilities, depending on whether the two circles on $P$ belong to the same annulus $A_{i}$.

If the two circles are on $A_{1}$, say, then one can use $D$ to isotop $t_{1}$ to the $\operatorname{arc} \beta=\partial D-A_{1}$ lying on the torus $\partial E\left(t_{2}\right)$, and there is an $\operatorname{arc} \alpha$ on $\Delta$ connecting the two ends of $\beta$ and is disjoint from the interior of $\beta$. Since $T$ is atoroidal, $t_{2}$ is a trivial arc, so by definition $T$ is a left torus tangle or a twist tangle.

If the two circles are on different $A_{i}$, the disk $D$ can be expanded to a disk $D^{\prime}$ in $B$ with $\partial D^{\prime}$ consisting of $t_{1}, t_{2}$ and two $\operatorname{arcs}$ on $\partial B$. Consider $D^{\prime}$ as a band in a 3 -ball. Since $T$ is atoroidal, the complement of the band is a solid torus, i.e the band is trivial in $B$. It is clear that in this case the tangle is a twist tangle.

Case (9). The intersection $\partial D \cap Q$ is a set of essential $\operatorname{arcs}$ on $Q$, which has four endpoints on $\partial Q \cap \partial P$, and has same number of points on the other two components of $\partial Q$. There are four possibilities as shown in Figure 2.4 (a) - (d). One can check that in subcase (a) $T$ is a 2-twist tangle, and in subcase (b) it is a 4-twist tangle. Subcase (c) cannot happen because then $\partial D$ would intersect one of the $A_{i}$ at least twice in the same direction, which would lead to a contradiction as in Case (1). We want to show that in subcase (d) $T$ is a left torus tangle. 


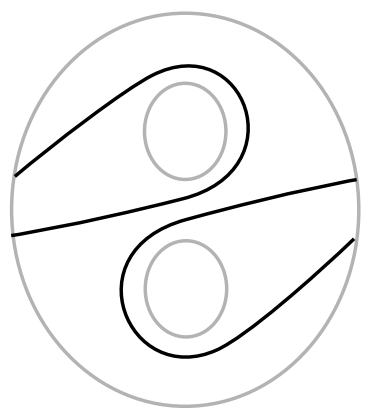

(a)

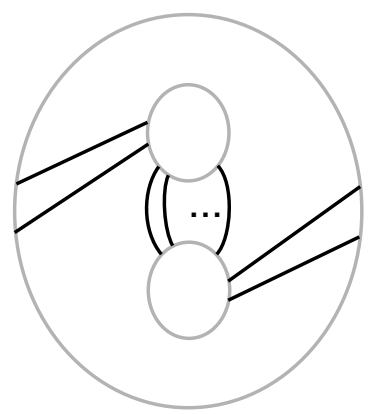

(c)

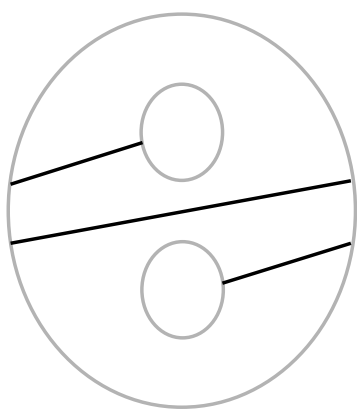

(b)

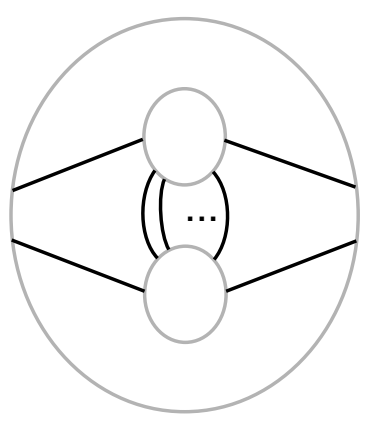

(d)

Figure 2.4

Let $A_{1}$ be the annulus with boundary on $Q$. Let $X=E\left(t_{1}\right)-\operatorname{Int} N(D)$. If $D$ is a nonseparating disk of $E\left(t_{1}\right)$, then $X$ is bounded by a sphere, so it is a 3-ball containing $t_{2}$. Since $X-\operatorname{Int} N\left(t_{2}\right)$ is bounded by a torus and is a subset of the atoroidal irreducible manifold $E$, it is a solid torus, that is, $t_{2}$ is an unknotted arc in $X$. If $D$ is separating, let $X_{1}, X_{2}$ be the two components of $X$. One of these, say $X_{1}$, is bounded by a sphere, so is a 3 -ball. Since $D$ is a compressing disk of $\partial E$, the string $t_{2}$ must be contained in $X_{1}$. The other component $X_{2}$ is now contained in $E$. Again by the property of $E$ we see that $X_{2}$ is a solid torus, and $t_{2}$ is a trivial arc in $X_{1}$. In either case, we conclude that $E\left(t_{1}\right)$ is a solid torus containing $t_{2}$ as a trivial arc. Let $\gamma$ be an arc on the gluing disk $\Delta$ connecting the two ends of $t_{2}$ and intersecting $\partial D$ twice. It remains to show that $t_{2}$ can be rel $\partial t_{2}$ isotoped to an $\operatorname{arc} \beta$ on $\partial E\left(t_{1}\right)$ disjoint from $\gamma$.

Let $D_{1}, D_{2}$ be the two copies of $D$ on $\partial X$. If $D$ is nonseparating, $X$ is a 3-ball. Since $\gamma$ intersects $\partial D$ twice, $\gamma$ is cut into three segments $\gamma_{1}, \gamma_{2}, \gamma_{3}$ on $\partial X$. Notice that each $\partial D_{i}$ intersects $\cup \gamma_{i}$ twice. Therefore when shrinking each $D_{i}$ to a point, $\cup \gamma_{i}$ is a 1-manifold on $\partial X$ with $\partial t_{2}$ as its boundary. Hence we can find a simple $\operatorname{arc} \beta$ on $\partial X$ connecting the two 
ends of $t_{2}$, so that $\beta$ is disjoint from $\left(\cup \gamma_{i}\right) \cup D_{1} \cup D_{2}$. Since $t_{2}$ is unknotted, it is isotopic to $\beta$ by an isotopy fixed on its endpoints. The corresponding isotopy in $E\left(t_{1}\right)$ moves $t_{2}$ to the $\operatorname{arc} \beta$ which is disjoint from $\gamma$. If $D$ is separating, we can consider the component $X_{1}$ instead, and get the required isotopy in a similar way.

\section{Nonsimple tangle sums}

Lemma 3.1 Suppose $(B, T)$ is an atoroidal tangle. Then the punctured sphere $P=\partial B-T$ is compressible if and only if $T$ is a rational tangle.

Proof. Cutting $B$ along a compressing disk $D$ of $P$ yields two balls $B_{1}$ and $B_{2}$, with each $B_{i}$ containing a single component $t_{i}$ of $T$. The boundary of $B_{i}-\operatorname{Int} N\left(t_{i}\right)$ is a torus, which must be compressible because $T$ is atoroidal. Therefore $t_{i}$ are unknotted in $B_{i}$.

Lemma 3.2 If $T=T_{1}+T_{2}$ is a nontrivial sum of atoroidal tangles, then $T$ is atoroidal.

Proof. The tangle space $E(T)$ is the union of $E\left(T_{1}\right)$ and $E\left(T_{2}\right)$ along a twice punctured disk $P$. Since the sum is nontrivial, it is easy to see that $P$ is incompressible in both $E\left(T_{i}\right)$. Let $F$ be a torus in $E(T)$, isotoped so that $F \cap P$ has minimal number of components. As $P$ is incompressible, $F \cap P$ consists of circles which are essential in both $P$ and $F$. Hence, each component of $F \cap E\left(T_{i}\right)$ is an essential annulus in $E\left(T_{i}\right)$ with boundary on $P$.

Since $T$ is a tangle, it has no circle component. Hence one of the tangles, say $T_{1}$, has the property that both strings have an end on the gluing disk $D$, so the homology map $H_{1}(P) \rightarrow H_{1}\left(E\left(T_{1}\right)\right)$ is an isomorphism. Let $A$ be a component of $F \cap E\left(T_{1}\right)$. By homological reason, the two boundary components of $A$ must be parallel on $P$, bounding an annulus $A^{\prime}$ on $P$. Since $T_{1}$ is atoroidal, the torus $A \cup A^{\prime}$ bounds a solid torus $V$. Notice that $A$ is compressible in the 3 -ball $B_{1}$, so $A$ must be longitudinal on $V$, otherwise $B_{1}$ would contain a punctured lens space. It follows that we can isotop $A$ through $V$ to reduce the number of components in $F \cap P$, a contradiction.

Lemma 3.3 Suppose $T=T_{1}+T_{2}$ is a nontrivial sum of atoroidal tangles. Then $T$ is a nontrivial tangle, and it is $\partial$-reducible if and only if, up to relabeling, $T_{1}$ is a 2-twist tangle, and $T_{2}$ is a rational tangle, in which case $E(T)$ is a handlebody.

Proof. Suppose $T_{1}$ is a 2 -twist tangle, and $T_{2}$ is a rational tangle. Let $P$ be the gluing

surface $\Delta-\operatorname{Int} N(T)$, let $\partial_{1}, \partial_{2}$ be the inner boundary components of $P$. Since $E\left(T_{1}\right)$ has a monogon, by compressing along it $E\left(T_{1}\right)$ becomes a product $A \times I$, where $A$ is an annulus, so that $E(T)$ is obtained from $E\left(T_{2}\right)$ by gluing $A \times 0$ to a neighborhood of $\partial_{1}$, and $A \times 1$ 
to that of $\partial_{2}$. Since $T_{2}$ is a rational tangle there is a compressing disk $D$ of $E\left(T_{2}\right)$ disjoint from $\partial_{1} \cup \partial_{2}$. Such a disk is disjoint from $A \times I$, so it gives rise to a compressing disk of $\partial E(T)$. Cutting along $D$ produces a manifold with each component bounded by a torus. Since $E(T)$ is atoroidal by Lemma 3.2, each of these components is a solid torus, so $E(T)$ is a handlebody. This proves the sufficiency.

Suppose that $T$ is $\partial$-reducible. Consider a compressing disk $D$ of $\partial E(T)$ which has minimal intersection with $P$. Since $P$ is incompressible, by innermost circle outermost arc arguments we may assume that $D \cap P$ consists of essential arcs in $P$. Let $\alpha$ be an arc of $D \cap P$ cutting off an outermost disk $D^{\prime}$ from $D$. Without loss of generality we may assume that $D^{\prime}$ lies in $E\left(T_{1}\right)$. Then $D^{\prime}$ is a monogon of $E\left(T_{1}\right)$. By Lemma 2.1, $T_{1}$ is a 2-twist tangle.

We now consider the other tangle $T_{2}$. Among all the components of $D \cap E\left(T_{2}\right)$, choose one, say $D^{\prime \prime}$, which is outermost on $D$. Let $\alpha_{1}, \ldots, \alpha_{k}$ and $\beta$ be the arcs of $\partial D^{\prime \prime} \cap P$, where the $\alpha_{i}$ 's are outermost arcs on $D$. There are at least one $\alpha_{i}$, for otherwise by the above argument $T_{2}$ would also be a 2 -twist tangle, so $T$ would contain a closed circle component, contradicting the definition of tangles. By the above argument, all the $\alpha_{i}$ have both endpoints on $\partial_{0}$. Since there is at least one $\alpha_{i}$, the arc $\beta$ cannot have one end on $\partial_{1}$ and the other on $\partial_{2}$. So it is either an arc with both ends on $\partial_{0}$, or it has one end on $\partial_{0}$, and the other on $\partial_{1}$, say. Let $A_{1}^{\prime}, A_{2}^{\prime}$ be the two annuli $N\left(T_{2}\right) \cap \partial E\left(T_{2}\right)$. Since $T$ has no circle components and $T_{1}$ is a 2-twist tangle, each $A_{i}^{\prime}$ must have exactly one boundary component on $P$. Hence if $\beta$ has both ends on $\partial_{0}$, then it is disjoint from $A_{1}^{\prime} \cup A_{2}^{\prime}$, so $\partial D^{\prime \prime}$ is a compressing disk of $\partial B_{2}-T_{2}$. By Lemma 3.1, this implies that $T_{2}$ is a rational tangle. If $\beta$ has one end on $\partial_{1}$, then $\partial D^{\prime \prime}$ intersects $A_{1}^{\prime}$ at a single essential arc, and is disjoint from $A_{2}^{\prime}$. Such a disk can be used to isotop the string $t_{1}^{\prime}$ of $T_{2}$ to an arc on $\partial B_{2}$ without crossing $t_{2}^{\prime}$, so again $\partial B_{2}-T_{2}$ is compressible, and $T_{2}$ is a rational tangle.

If $T$ is a trivial tangle, there is a compressing disk $D$ of $\partial E(T)$ disjoint from $\partial N(T)$. But from the proof above we know that $D \cap E\left(T_{i}\right)$ contains a monogon which intersects $\partial N\left(T_{i}\right)$.

Lemma 3.4 Let $T=T_{1}+T_{2}$ be a nontrivial sum of atoroidal tangles. Suppose $E(T)$ contains an essential annulus $Q$. Then up to relabeling of $T_{i}$ one of the following holds:

(i) $T_{1}$ is $\Delta$-annular, and $Q$ can be isotoped to a $\Delta$-essential annulus in $E\left(T_{1}\right)$.

(ii) $T_{1}$ is a q-twist tangle with $q$ odd, and $T_{2}$ is either a left torus tangle or a p-twist tangle with $|p| \geq 3$.

(iii) Both $T_{i}$ are wrapping tangles, and the unknotted string of $T_{1}$ is glued to that of $T_{2}$. Moreover, in cases (ii) and (iii), $Q$ intersect each $T_{i}$ in bigons. 
Proof. Isotop $Q$ in $E(T)$ so that the number of components in $P \cap Q$ is minimal, where $P=E\left(T_{1}\right) \cap E\left(T_{2}\right)$. If $Q$ is disjoint from $P$, then up to relabeling we may assume that $Q$ is in $E\left(T_{1}\right)$, and one can see that it is $\Delta$-essential in $E\left(T_{1}\right)$, so (i) follows. Thus we may assume that $P \cap Q \neq \emptyset$. Since $Q$ is essential, by an innermost circle outermost arc argument we may assume that $P \cap Q$ consists of essential arcs and circles on $P$. As $P$ is incompressible, we may also assume that each circle component of $P \cap Q$ is essential on $Q$.

Case (1). $P \cap Q$ consists of essential circles only.

Since $T$ is a tangle, one of the $T_{i}$, say $T_{1}$, has the property that each string has exactly one endpoint on $\Delta$. Consider a component $A$ of $Q \cap E\left(T_{1}\right)$, an annulus with one or both boundaries on $P$. Since $Q$ is disjoint from $\partial P, \partial A$ lies on the sphere $\partial B_{1}$, so $\partial A$ bounds an annulus $A^{\prime}$ on $\partial E\left(T_{1}\right)$. The torus $A \cup A^{\prime}$ bounds a solid torus $V$ in $E\left(T_{1}\right)$ because $E\left(T_{1}\right)$ is irreducible and atoroidal. Since $A$ is compressible in $B_{1}$, for homological reason $A$ must run once along the longitude of $V$. Therefore $A$ is parallel to $A^{\prime}$, so we can isotop $A$ off $P$ to reduce the number of components in $P \cap Q$, a contradiction.

Case (2). $P \cap Q$ are arcs which are essential on both $P$ and $Q$.

Then $P$ cuts $Q$ into bigons of $E\left(T_{i}\right)$. By Lemma 2.2, they are not of types (1) - (3). If none of the bigons are of types (4)-(6), then $E\left(T_{i}\right)$ are twist tangles or left torus tangles. But they cannot both be left torus tangles, otherwise $T$ would have a closed circle. Also, $T_{i}$ cannot be 2-twist tangle, for then $E(T)$ would be a handlebody (by Lemma 3.3) if the other $T_{j}$ is a twist tangle, and $T$ would have a closed circle component if $T_{j}$ is a torus tangle. Therefore conclusion (ii) follows. Suppose one of the bigons $D$ is of types (4)-(6). Such a bigon is characterized by the fact that one of the arcs of $\partial D \cap P$ has both ends on the inner circles of $P$. Hence, if $D$ lies in $E\left(T_{1}\right)$, then the bigon in $E\left(T_{2}\right)$ which intersects $D$ at the above mentioned arc is of type (4)-(6) also. Therefore both $T_{i}$ are wrapping tangles. If $t_{1}$ is a component of $T_{1}$, by the proof of Lemma 2.2 we see that $t_{1}$ is the unknotted arc if and only if $D \cap P$ has a component $\alpha$ with both ends on $\partial_{1}$ or one end on $\partial_{1}$ and the other on $\partial_{0}$, where $\partial_{1}$ is the circle lying on $\partial N\left(t_{1}\right)$. Therefore the knotted arc of $T_{1}$ is connected to the knotted arc of $T_{2}$. Conclusion (iii) follows.

Case (3). $P \cap Q$ contains inessential arcs of $Q$ but no essential arcs.

An outermost inessential arc on $Q$ cuts off a disk which is a monogon of one of the tangles, say $T_{1}$. By Lemma $2.1 T_{1}$ is a 2-twist tangle. We claim that all arcs of $P \cap Q$ are outermost on $Q$. If not, choose an $\alpha$ which cuts off a disk $D$ containing some outermost arcs, and outermost arcs only, in its interior. $D \cap E\left(T_{2}\right)$ is a compressing disk of $\partial E\left(T_{2}\right)$ which intersects $\partial N\left(T_{2}\right)$ at most once, because $\alpha$ is the only arc on $D$ which may have an end on the inner circles of $P$. By the argument of Lemma 3.3 we see that $T_{2}$ is a rational tangle. But then by Lemma $3.3 E(T)$ is a handlebody, which could not contain any essential 
annulus, contradicting the assumption.

Hence, a component $A$ of $Q \cap E\left(T_{2}\right)$ is an annulus with $\partial A$ lying on $\partial B_{2}$. As in case (1), $A$ is boundary parallel in $E\left(T_{2}\right)$, which implies that either $A$ can be isotoped off $E\left(T_{2}\right)$, or $Q$ is $\partial$-compressible. Neither case is possible.

Case (4). $P \cap Q$ contains both essential arcs and inessential arcs on $Q$.

Since all inessential arcs are outermost as we have shown in case (3), a component $D$ of $Q \cap E\left(T_{2}\right)$ is a disk such that $\partial D$ contains two essential arcs $\alpha_{1}, \alpha_{2}$ and some inessential $\operatorname{arcs}$ of $Q$. In general $D$ is not a bigon, but the two essential arcs lie on $P$ in one of the type shown on Figure 2.1. Since $P \cap Q$ contains some inessential arcs, which have both ends on the outer circle of $P$, we see that type (2)-(6) do not happen. Type (1) can be ruled out similarly as in Lemma 2.2. In all the remaining cases, $\partial D$ intersects each of the inner circle, and hence each of the annuli in $\partial N\left(T_{2}\right)$, at most once. By the arguments of Lemma 3.3 and Case (8) of Lemma 2.2, the tangle $T_{2}$ is a rational tangle, so by Lemma $3.3 E(T)$ is a handlebody and contains no essential annuli.

Lemma 3.5 The converse of Lemma 3.4 is true. That is, if (i), (ii), or (iii) in Lemma 3.4 holds, then $E(T)$ contains an essential annulus.

Proof. First assume (i) of Lemma 3.4 holds, and let $A$ be a $\Delta$-essential annulus in $E\left(T_{1}\right)$. We will show that $A$ is essential in $E(T)$. As before, let $P=E\left(T_{1}\right) \cap E\left(T_{2}\right)=\Delta \cap E\left(T_{1}\right)$. Suppose $D$ is a $\partial$-compressing disk of $A$. By definition of $\Delta$-essential annulus, we must have $D \cap P \neq \emptyset$. As the sum is nontrivial, $P$ is incompressible in $E(T)$, so we may assume that $D \cap P$ is a set of essential arcs in $P$. There is at least one disk (an outermost disk) $D^{\prime}$ in $D$ with interior disjoint from $P$, such that $\partial D^{\prime}$ consists of an arc of $D \cap P$ and an arc on $\partial D-A$. By definition such a disk is a monogon, so by Lemma 2.1 one of the $T_{i}$ is a 2 -twist tangle. It must be $T_{2}$, because by Lemma 4.4 a 2 -twist tangle is $\Delta$-anannular. But by Lemma 4.1(i) the two ends of $\Delta \cap T_{1}$ belong to the same string of $T_{1}$, which is a contradiction because then $T$ would have a closed circle component.

Now consider (ii) and (iii) of Lemma 3.4. From the proof of Lemma 2.2 one can see that if $\left(B_{i}, T_{i}, \Delta\right)$ is a twist tangle or left torus tangle there is a bigon $D_{i}$ such that $D_{i} \cap \Delta$ is the arcs in Figure 2.1(8), and if $\left(B_{i}, T_{i}, \Delta\right)$ is a wrapping tangle there is a bigon $D_{i}$ with $D_{i} \cap \Delta$ the arcs in Figure 2.1(4). Call these the preferred bigons. In case (ii) or (iii), we glue the two preferred bigons $D_{1}, D_{2}$ together to form a surface $Q$. If $Q$ is an annulus, let $A=Q$. If $Q$ is a Möbius band, let $A$ be the frontier of $N(Q)$ in $E(T)$.

Since $\partial Q$ intersects $\partial_{2}$ at one point, each component of $\partial A$ intersects $\partial_{2}$ once, so it is an essential curve on $\partial E(T)$. If $A$ is compressible in $E(T)$, each component of $\partial A$ bounds a disk in $E(T)$, therefore $\partial E(T)$ is compressible. Now suppose $A$ is $\partial$-compressible. If $Q$ is an 
annulus, a $\partial$-compression of $A$ produces a disk $D$ with $\partial D$ intersects $\partial_{2}$ an odd number of points, so again $\partial E(T)$ is compressible. If $Q$ is a Möbius band and $A$ has a $\partial$-compressing disk $D$, then $N(Q) \cup N(D)$ is a solid torus submanifold of $E(T)$ whose frontier is a disk. Since $\partial E(T)$ has genus 2, this disk is a compressing disk of $\partial E(T)$. In all cases we have shown that $\partial E(T)$ is compressible. By Lemma 3.3, one of $T_{i}$ is a 2-twist tangle. Since none of the tangles in (ii) or (iii) is such, we get a contradiction.

Theorem 3.6 Suppose $T=T_{1}+T_{2}$ is a nontrivial sum of atoroidal tangles. Then $T$ is nonsimple if and only if, up to relabeling of $T_{i}$, one of the following holds.

(1) $T_{1}$ is a 2-twist tangle, and $T_{2}$ is a rational tangle;

(2) Both $T_{i}$ are twist tangles;

(3) Both $T_{i}$ are wrapping tangles, and the unknotted string of $T_{1}$ is glued to the unknotted string of $T_{2}$;

(4) $T_{1}$ is $\Delta$-annular.

Proof. In case (1), by Lemma $3.3 T$ is $\partial$-reducible. In case (2), (3) or (4), by Lemma 3.5 $E(T)$ contains an essential annulus unless $T_{2}$ in case (2) is a 2-twist tangle, which has been covered by (1). This proves sufficiency. Now suppose $T$ is nonsimple. As a tangle space $E(T)$ is irreducible. By Lemma 3.2 it is also atoroidal. Hence $E(T)$ is either $\partial$-reducible or annular. In the first case by Lemma 3.3 the conclusion (1) holds. In the second case by Lemma 3.4 one of the conclusion (i), (ii) or (iii) of Lemma 3.4 holds. These are covered by $(2)$ - (4) here, except when $T_{2}$ in (ii) of Lemma 3.4 is a left torus tangle. Here one is referred to the last paragraph of the proof of Lemma 4.3, which shows that a left torus tangle is $\Delta$-annular, hence in this case (4) holds after relabeling of $T_{1}$ and $T_{2}$.

\section{$4 \Delta$-annular algebraic tangles}

The following lemma gives some basic properties of $\Delta$-annular tangles.

Lemma 4.1 Suppose $(B, T, \Delta)$ is a nontrivial atoroidal tangle containing a $\Delta$-essential annulus $A$. Let $P=\Delta \cap E(T), A_{i}=\partial N\left(t_{i}\right) \cap \partial E(T)$, and $P^{\prime}=(\partial B \cap E(T))-$ IntP. Then

(i) One of the $A_{i}$, say $A_{1}$, has both boundary components contained in P;

(ii) $A$ is disjoint from $A_{1}$;

(iii) $A$ can not be isotoped so that $\partial A$ is disjoint from $m_{2}$, where $m_{2}$ is a meridian of $t_{2}$, i.e. an essential loop on $A_{2}$;

(iv) If no component of $\partial A$ is parallel to $\partial \Delta$, then $\partial A$ bounds an annulus $A^{\prime}$ on $P^{\prime} \cup A_{2}$, such that $A \cup A^{\prime}$ bounds a solid torus $V$, and $A$ runs at least twice around the longitude of $V$. 
(v) If $\partial A=\partial_{1} \cup \partial_{2}$ has one component $\partial_{1}$ parallel to $\partial \Delta$, then $A$ is an essential annulus in $E(T)$.

(vi) If $E(T)$ is $\partial$-irreducible, then any $\Delta$-essential annulus is essential.

Proof. (i). Assume that both $A_{i}$ intersect $\Delta$. Then $P^{\prime} \cup A_{1} \cup A_{2}$ is isotopic to $P^{\prime}$, so by an isotopy we may assume that $\partial A \subset P^{\prime}$. In this case the natural map $H_{1}\left(P^{\prime}\right) \rightarrow H_{1}(E(T))$ is an isomorphism. Thus the two components of $\partial A$ are parallel on $P^{\prime}$, hence bound an annulus $A^{\prime}$ on $P^{\prime}$. Since $E(T)$ is atoroidal, $A \cup A^{\prime}$ bounds a solid torus $V$ in $E(T)$. $A$ can not be meridional on $\partial V$, because it is incompressible in $E(T)$. $A$ can not be longitudinal either, otherwise $A$ is rel $\partial A$ isotopic to $A^{\prime}$, contradicting the assumption that it is $\Delta$-essential. Hence $A$ runs at least twice along the longitude of $V$. Since $\partial A$ lies on $\partial B, A$ bounds a two handle $H$ in $B-V$. But then $V \cup H$ would be a punctured lens space in the 3 -ball $B$, which is absurd.

(ii). $H_{1}(E(T))$ is generated by $m_{1}$ and $m_{2}$, where $m_{i}$ is an essential loop on $A_{i}$. Thus none of the curves on $P^{\prime} \cup A_{2}$ is homologous to $m_{1}$. It follows that either $\partial A \subset A_{1}$, or $\partial A \subset P^{\prime} \cup A_{2}$. If $\partial A \subset A_{1}$, one can use the above argument to show that there would be a punctured lens space in $B$.

(iii). Now $\partial A$ is contained in $P^{\prime} \cup A_{2}$. If after some isotopy $\partial A$ is disjoint from $m_{2}$, then $\partial A$ can be isotoped into $P^{\prime}$. For homological reasons $\partial A$ can not have one component parallel to $\partial \Delta$ while the other parallel to $m_{2}$. Therefore, after isotoping a component of $\partial A$ through $A_{2}$ if necessary, we may assume that $\partial A$ are parallel circles on $P^{\prime}$. By the proof of (i) this is impossible.

(iv). $P^{\prime} \cup A_{2}$ is a once punctured torus, so if no component of $\partial A$ is parallel to $\partial \Delta$, then $\partial A$ bounds an annulus $A^{\prime}$ on $P^{\prime} \cup A_{2}$. As in the proof of (i), one can show that $A \cup A^{\prime}$ bounds a solid torus $V$, and $A$ is neither meridional nor longitudinal on $\partial V$.

(v). A $\partial$-compressing disk of $A$ has to intersect both components of $\partial A$. Since $\partial_{1}$ separates $\partial_{2}$ from $P$, no $\partial$-compressing disk of $A$ could intersect $P$.

(vi). By (v) and (iv), we may assume that the two components of $\partial A$ bound an annulus $A^{\prime}$ on $\partial E(T)$. Let $D$ be a $\partial$-compressing disk of $A$. Since $A$ is not parallel to $A^{\prime}, D \cap A^{\prime}=\emptyset$. Therefore, after surgering $A$ along $D$, we get a disk $D^{\prime}$ properly embedded in $E(T)$ whose boundary is essential on $\partial E(T)$.

There are many $\Delta$-annular tangles. The following lemma gives some sufficient conditions, which are easy to check in practice.

Lemma 4.2 A nontrivial atoroidal tangle $(B, T, \Delta)$ with $T=t_{1} \cup t_{2}$ is $\Delta$-annular if it satisfies the following conditions. 
(1) $t_{1}$ has both ends on $\Delta$;

(2) there is a solid torus $V$ in $B$ disjoint from $t_{1}$, such that $V \cap \partial B$ is a disk $\Delta^{\prime}$ in $\partial B-\Delta$

(3) $t_{2}$ lies on $\partial V$, so that if $D$ is a meridional disk of $V$ disjoint from $\partial B$, then $t_{2}$ intersect $\partial D$ algebraically at least twice.

Proof. The punctured torus $\partial V-\operatorname{Int} \Delta^{\prime}$ intersects $E(T)$ in an annulus $A$ disjoint from $\Delta$. Condition (3) implies that $A$ runs around $V$ at least twice. So $A$ must be incompressible in $E(T)$, for otherwise the union of $V$ with a regular neighborhood of a compressing disk of $A$ would be a punctured lens space in $B$, which is impossible. Therefore, we need only show that all $\partial$-compressing disks of $A$ intersects $\Delta$.

Let $V^{\prime}=V \cap E(T)$. Then $\partial A$ bounds an annulus $A^{\prime}=V^{\prime} \cap \partial E(T)$, and $A \cup A^{\prime}=\partial V^{\prime}$. Assume $A$ is $\partial$-compressible and let $D$ be a $\partial$-compressing disk. Since $A$ run more than once around $V^{\prime}, \partial D$ can not be contained in $A \cup A^{\prime}$. Therefore, after surgery along $D, A$ becomes a compressing disk $Q$ of $\partial E(T)$. Note that $\partial Q$ bounds a once punctured torus $F$ on $\partial E(T)$, which is the union of $A^{\prime}$ with a regular neighborhood of the arc $\partial D \cap \partial E(T)$. If $\partial D$ is disjoint from $\Delta$, then $F$ lies in $\partial E(T)-\Delta$, so $\partial D=\partial F$ is parallel to $\partial \Delta$, which implies that $Q$ is a compressing disk of $\partial B-T$, contradicting Lemma 3.1. Therefore, $A$ is a $\Delta$-essential annulus.

Lemma 4.3 Any $(B, T, \Delta)$ in the set $\mathcal{S}$ is $\Delta$-annular.

Proof. After an isotopy, the tangles $R\left[r_{1}, r_{2} ; r_{3}\right]$ in (1) - (5) of the definition of $\mathcal{S}$ can be drawn as in Figure 4.1.

For all of them except the last one, it is easy to find a solid torus $V$ satisfying the conditions of Lemma 4.2. Figure 4.2(a) illustrates a solid torus for the tangle $R\left[\frac{2}{3},-\frac{1}{3} ;-\frac{1}{3}\right]$. One can also find a torus if $T$ is a $2 n$-twist tangle with $|n| \geq 2$. Hence $T$ is $\Delta$-annular by Lemma 4.2. For the tangle $R\left[\frac{1}{3},-\frac{1}{3} ; 0\right]$, an annulus is shown in Figure $4.2(\mathrm{~b})$. It has one boundary component parallel to $\partial \Delta$, so it is incompressible. Since its two boundary components are not parallel, if it is $\partial$-compressible, then $\partial E(T)$ would be compressible, which is impossible by Lemma 3.3 .

We need to show that if $T$ is similar to one of the above, then it is also $\Delta$-annular. Since $(B, T ; \Delta)=R\left[-r_{1},-r_{2} ;-r_{3}\right]$ is the mirror image of $\left(B^{\prime}, T^{\prime} ; \Delta^{\prime}\right)=R\left[r_{1}, r_{2} ; r_{3}\right]$, there is an orientation reversing map sending $E(T)$ to $E\left(T^{\prime}\right)$ which maps $\Delta \cap E(T)$ to $\Delta^{\prime} \cap E\left(T^{\prime}\right)$. Hence $T$ is $\Delta$-annular if and only if $T^{\prime}$ is. Now consider $R\left[r_{2}, r_{1} ; r_{3}\right]$. If $R\left[r_{2}, r_{1} ; r_{3}\right]$ is not $R\left[\frac{1}{q}, \frac{1}{2} ;-\frac{1}{2}\right]$ or $R\left[-\frac{1}{3}, \frac{1}{2} ;-\frac{1}{4}\right]$, one can prove that $R\left[r_{2}, r_{1} ; r_{3}\right]$ is $\Delta$-annular by finding a solid torus above. Notice that $R\left[\frac{1}{q}, \frac{1}{2} ;-\frac{1}{2}\right]$ is a $(2, q)$ left torus tangle, and $R\left[-\frac{1}{3}, \frac{1}{2} ;-\frac{1}{4}\right]$ is a $(3,2)$ left torus tangle, so it remains to show that a left torus tangle is $\Delta$-annular. 

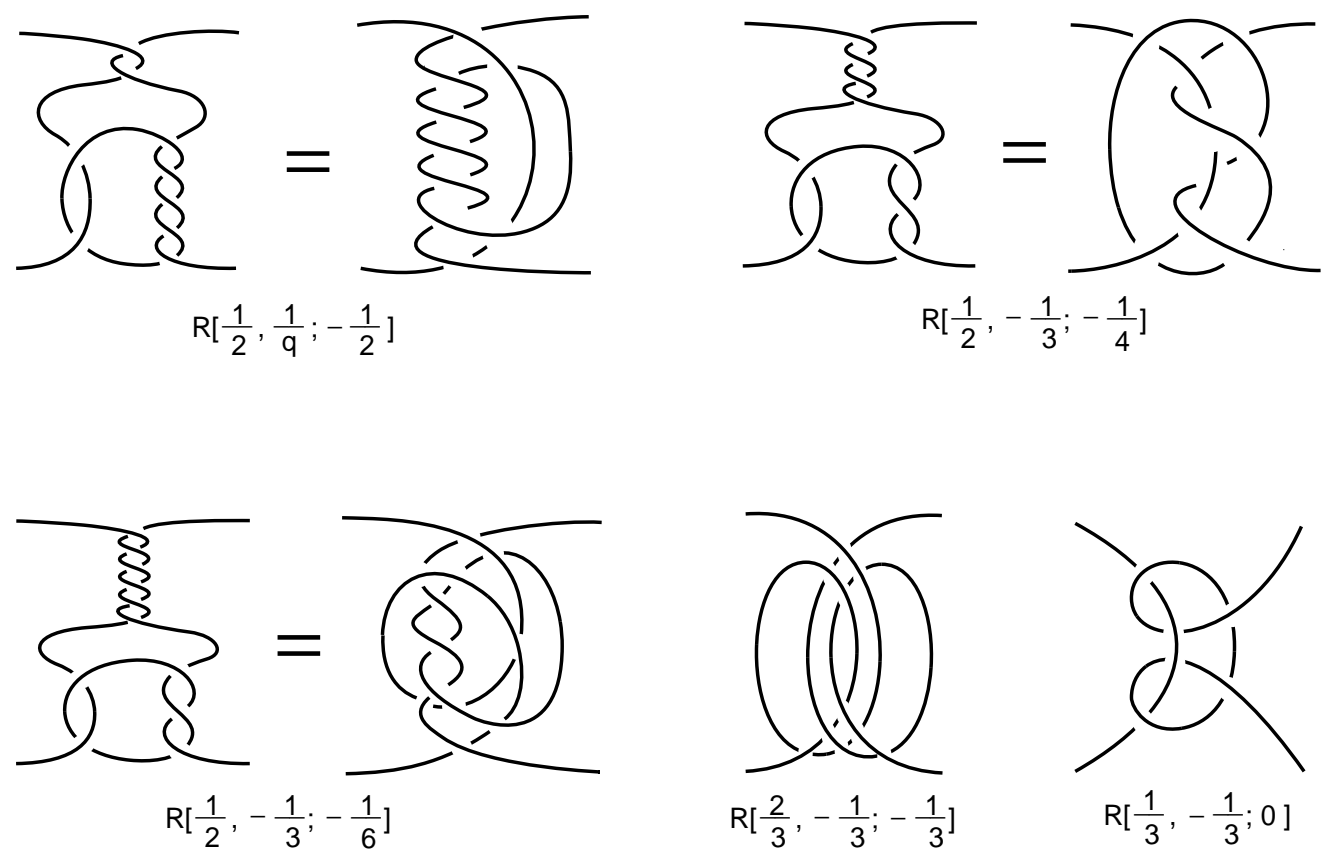

Figure 4.2
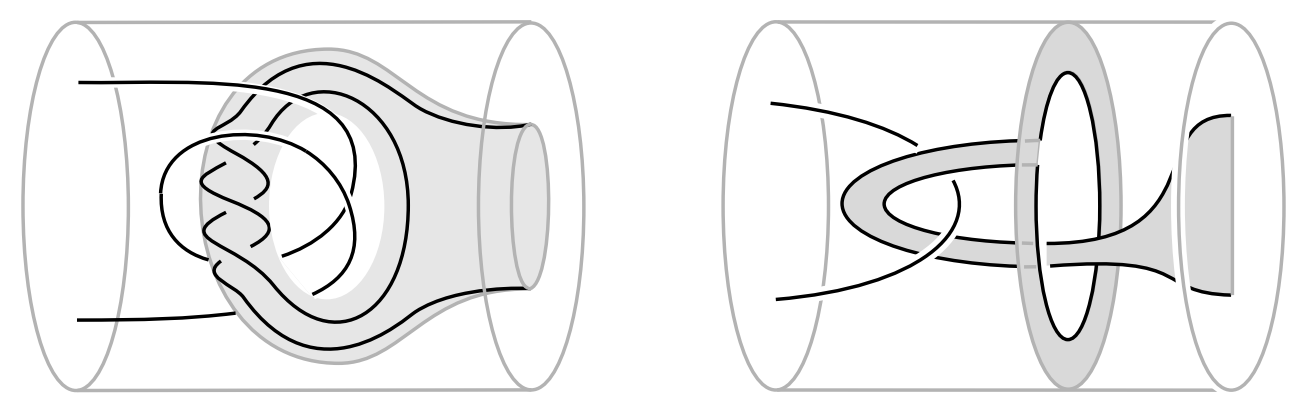

Figure 4.2

Recall from the definition that for a $(p, q)$ left torus tangle $(B, T, \Delta)$ with $T=t_{1} \cup t_{2}$, $E\left(t_{1}\right)$ is a solid torus $V, \Delta$ is a disk on $\partial V$, and $t_{2}$ is a string isotopic to some arc $\beta$ on $\partial V$, and there is an arc $\alpha$ on $\Delta$ such that $\alpha \cup \beta$ is a simple closed curve running $p$ times around $V$. By definition of torus tangles, we have $|p| \geq 2$. Let $A$ be the frontier of a regular neighborhood of $\alpha \cup \beta$ which contains $t_{2} \cup \Delta$. Then $A$ is a $\Delta$-essential annulus.

Suppose $(B, T, \Delta)$ is $\Delta$-annular, with $A$ a $\Delta$-essential annulus. By Lemma 4.1(i) we may assume that $\partial t_{1} \subset \Delta$. Consider $B$ as a 3 -ball in $S^{3}$. Connecting the ends of $t_{1}$ (resp. 
$\left.t_{2}\right)$ by an arc in $\Delta($ resp. $\partial B-\Delta)$, we obtain a link $L=l_{1} \cup l_{2}$, where $l_{i} \supset t_{i}$. Call $L$ the induced link of $(B, T, \Delta)$. Clearly, the link exterior $E(L)$ can be obtained by attaching a 2-handle to $E(T)$ along $\partial \Delta$.

Lemma 4.4 A nontrivial rational tangle $(B, T, \Delta)=M[p / q]$ is $\Delta$-annular if and only if it is a (2n)-twist tangle with $|n| \geq 2$.

Proof. By our definition, $M\left[r_{1}\right]$ is equivalent to $M\left[r_{2}\right]$ if and only if $r_{1}$ is equivalent to $r_{2}$ $\bmod \mathbf{Z}$, so we may assume that $1 \leq|p|<q$. The induced link $L$ is a 2-bridge link associated to the same rational number $p / q$, which we denote by $L(p / q)$. Let $A^{\prime}$ and $V$ be as in Lemma 4.1(iv), now considered as in $E(L)=E(T) \cup H$, where $H$ is a 2-handle attached to $E(T)$ along $\partial \Delta$. Let $M$ be the manifold obtained by Dehn filling on $E(L)$ along the central curve of $A^{\prime}$. Then $A^{\prime}$ bounds a 2-handle $H^{\prime}$ in the attached solid torus. Hence $M$ contains the punctured lens space $V \cup H^{\prime}$, so it is reducible.

Consider $l_{2}$ as a knot in the solid torus $W=E\left(l_{1}\right)=S^{3}-\operatorname{Int} N\left(l_{1}\right)$. Since $p / q \neq 1 / 0$, $L$ is not a trivial link, so $\partial W$ is incompressible in $E(L)$. As the reducible manifold $M$ is obtained from $W$ by Dehn surgery on $l_{2}$, by [11] $l_{2}$ is a cable knot in $W$. Since $L$ is a 2-bridge link, there is no essential torus in $E(L)$ (see the proof of [6, Theorem 1(a)]). Thus $l_{2}$ is isotopic to some $(r, s)$ knot on $\partial W$ with $s>1$, i.e. it runs $r$ times along the longitude and $s$ times along the meridian of $l_{1}$. However, as a component of a 2-bridge link, $l_{2}$ is a trivial knot in $S^{3}$, so we must have $r= \pm 1$. It follows that $L$ is a $(2,2 n)$ torus $\operatorname{link}(n= \pm s)$, which is a 2-bridge link associated to the rational number $1 / 2 n$. By the classification of 2-bridge links (see [3, Theorem 12.6]), $p / q=1 / 2 n$, so $(B, T, \Delta)$ is a $( \pm 2 n)$-twist tangle. Since $|n|=s \geq 2$, the result follows.

If $(B, T)$ is a tangle, we can glue the left disk to the right disk by the reflection along the plane containing $C_{y}$. The image of $T$ is a link in $S^{3}$. When $T$ is the Montesinos tangle $M\left(r_{1}, \ldots, r_{n}\right)$, the link so obtained is called a Montesinos link, denoted by $L\left(r_{1}, \ldots r_{n}\right)$. We refer the reader to [3] for classifications of Montesinos links. We need the following lemma, which is a consequence of a result of Bonahon and Siebenmann [2, Theorem A8].

Lemma 4.5 If $L=L\left(r_{1}, r_{2}, r_{3}\right)$ ( $r_{i}$ are non-integral rational numbers) is a two component Montesinos link such that $E(L)$ is a Seifert fiber space, then $L$ is either $L\left(\frac{1}{2}, \frac{1}{q},-\frac{1}{2}\right)$ or $L\left( \pm \frac{1}{2}, \mp \frac{1}{3}, \mp \frac{1}{4}\right)$.

Here is the idea of the proof of Lemma 4.5. The double branch cover $X$ of $S^{3}$ branched over $L$ has a natural Seifert fibration with 3 singular fibers. If $E(L)$ is a Seifert fiber space, then $X$ has another fibration in which the lift of $L$ are fibers. There are only a few manifolds 
which have different Seifert fibrations. The result then follows by calculating the Seifert invariants of $X$. See [2] for details.

Lemma 4.6 Suppose $(B, T, \Delta)$ is $\Delta$-annular, and suppose the tangle $(B, T)$ is $M\left(\frac{1}{2}, \frac{p}{q}\right)$. Then $(B, T, \Delta)$ is similar to one of the $R\left[\frac{1}{2}, \frac{1}{q},-\frac{1}{2}\right], R\left[\frac{1}{2},-\frac{1}{3},-\frac{1}{4}\right]$ or $R\left[\frac{1}{2},-\frac{1}{3},-\frac{1}{6}\right]$.

Proof. By assumption $(B, T)=\left(B_{1}, T_{1}, \Delta_{1}\right)+\left(B_{2}, T_{2}, \Delta_{2}\right)$. We claim that $\partial \Delta_{1}$ is not parallel to $\partial \Delta$ on the punctured sphere $\partial B-T$.

Since $T_{1}$ is a 2 -twist tangle, $T_{2}$ can not be a $(2 n)$-twist tangle, otherwise $T$ would have a circle component. Thus by Lemma 4.2 , both $T_{i}$ are $\Delta_{i}$-anannular, so $A$ can not be isotoped to be disjoint from $\Delta_{1}=\Delta_{2}$. On the other hand, if $\partial \Delta_{i}$ is parallel to $\partial \Delta$, then $A$ can be isotoped to be disjoint from $\partial \Delta_{i}$. Let $P=\Delta \cap E(T)$. By Lemma 4.1(iii), $A$ has to intersect the inner boundaries of $P$, so $A \cap P$ has some arc components. Thus some components of $A \cap E\left(T_{i}\right)$ are monogons or bigons disjoint from the outer boundary of $P$. But by Lemma 2.1 and 2.2, this can not happen. This proves the claim.

Let $L=l_{1} \cup l_{2} \subset S^{3}$ be the induced link of $(B, T, \Delta)$. Clearly, one component, say $l_{1}$, is a trivial knot in $S^{3}$, and the other component $l_{2}$ is a $p / q$ 2-bridge knot. $\left(S^{3}, L\right)$ can be obtained by taking the union of $(B, T)$ with a trivial tangle $\left(B^{\prime}, T^{\prime}\right)$, so that $\partial \Delta$ bounds a disk in $B^{\prime}-T^{\prime}$. Hence, $L$ is actually a Montesinos $\operatorname{knot} L\left(\frac{1}{2}, \frac{p}{q}, \frac{p^{\prime}}{q^{\prime}}\right)$ for some $p^{\prime} / q^{\prime}$. The above claim means that $\partial \Delta_{1}$ does not bound disk in $B^{\prime}-T^{\prime}$, so $q^{\prime} \neq 0$. In particular, $E(L)$ is irreducible [9].

Since $E(T)$ is a handlebody (Lemma 3.3), by Lemma $4.1(\mathrm{v}) \partial A$ has no component parallel to $\partial \Delta$, so we can apply Lemma 4.1(iv). Let $A^{\prime}$ and $V$ be the annulus and torus given there. Let $\partial_{1}, \partial_{2}$ be the components of $\partial N(L)$. We may assume that $A^{\prime}$ is on $\partial_{1}$. Let $A^{\prime \prime}=\partial_{1}-\operatorname{Int} A^{\prime}$. Pushing $A \cup A^{\prime \prime}$ into $E(L)$, we obtain a torus $S$. It separates the two component of $\partial E(L)$. Since $E(L)$ is irreducible, $S$ must be incompressible. $S$ cuts $E(L)$ into two pieces. Since $A$ runs at least twice around the longitude of $\partial V$ (Lemma 4.1(iv)), the component of $E(L)-\operatorname{Int} N(S)$ containing $\partial_{1}$ is a cable space (i.e. the exterior of a knot $K$ in a solid torus $W$ such that $K$ lies on a torus parallel to $\partial W$ and $K$ runs at least twice around $W)$. In particular, $S$ is not parallel to $\partial_{1}$. Therefore, either $S$ is essential in $E(L)$, or it is parallel to $\partial_{2}$. We separate the two cases.

CASE 1. ( $S$ is essential.)

Up to reflection there are four Montesinos links whose complement contains some essential torus, only one of which has two components, and has a 2-twist tangle as one of its rational tangles (see [9]). It is the link $L\left(\frac{1}{2},-\frac{1}{3},-\frac{1}{6}\right)$. Since the sum of a 2 -twist tangle with a 6 -twist tangle has a closed circle component, it follows that $T_{2}$ must be a (-3)-twist tangle. Thus the tangle $(B, T)$ is the one inside the rectangle (which represents $\partial B$ ) of Figure 4.3(a). 
The disk $\Delta$ is determined by the property that $\partial \Delta$ bounds a disk in the (-6)-twist tangle. By an isotopy of the triple $\left(S^{3}, \partial B, L\right)$ we get Figure $4.3(\mathrm{~b})$. It is now clear that $(B, T ; \Delta)$ is either $R\left[\frac{1}{2},-\frac{1}{3} ;-\frac{1}{6}\right]$ or $R\left[\frac{1}{3},-\frac{1}{2} ;-\frac{1}{6}\right]$. If the link is $L\left(-\frac{1}{2}, \frac{1}{3}, \frac{1}{6}\right)$, the corresponding tangle is the reflection of the above tangles. They are all similar to $R\left[\frac{1}{2},-\frac{1}{3} ;-\frac{1}{6}\right]$.
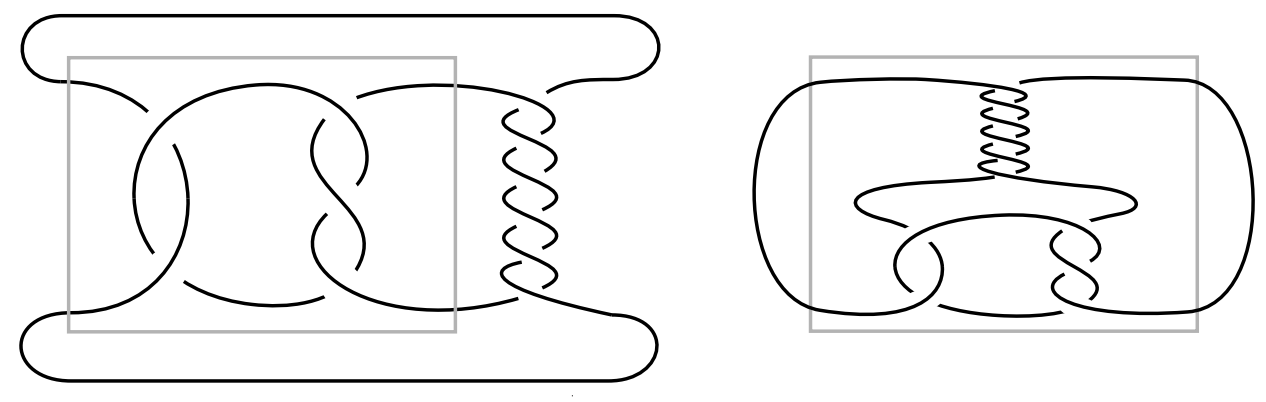

Figure 4.3

CASE 2. ( $S$ is parallel to $\partial_{2}$.)

In this case $E(L)$ is a cable space, so it is a Seifert fiber space. By Lemma $4.5, L$ is one of the $L\left(\frac{1}{2}, \frac{1}{q},-\frac{1}{2}\right)$ or $L\left( \pm \frac{1}{2}, \mp \frac{1}{3}, \mp \frac{1}{4}\right)$. With the same method as in Case 1 , one can see that $(B, T ; \Delta)$ is a tangle similar to $R\left[\frac{1}{2}, \frac{1}{q} ;-\frac{1}{2}\right]$ or $R\left[\frac{1}{2},-\frac{1}{3} ;-\frac{1}{4}\right]$.

Proposition $4.7 A$ marked algebraic tangle $(B, T, \Delta)$ is $\Delta$-annular if and only if either it is in $\mathcal{S}$, or $(B, T)$ is a nontrivial sum $\left(B_{1}, T_{1}, \Delta_{1}\right)+\left(B_{2}, T_{2}, \Delta_{2}\right)$, such that $\Delta \subset \partial B_{2}$, and $\left(B_{1}, T_{1}, \Delta_{1}\right) \in \mathcal{S}$.

Proof. If $(B, T, \Delta)$ is in $\mathcal{S}$ then by Lemma 4.3 it is $\Delta$-annular. If $(B, T)=\left(B_{1}, T_{1}, \Delta_{1}\right)+$ $\left(B_{2}, T_{2}, \Delta_{2}\right), \Delta \subset \partial B_{2}$, and $\left(B_{1}, T_{1}, \Delta_{1}\right) \in \mathcal{S}$, then by Lemma 3.5 a $\Delta_{1}$-essential annulus $A$ is essential in $E(T)$. Since $A$ is disjoint from $\Delta$, it is a $\Delta$-essential annulus of $(B, T, \Delta)$. Thus the conditions are sufficient.

Now assume $(B, T, \Delta)$ is $\Delta$-annular. Let $A$ be a $\Delta$-essential annulus in $E(T)$. If $T$ is either rational or a sum of a 2-twist tangle and a rational tangle, we are done by Lemma 4.4 and Lemma 4.6. So we may assume that $(B, T)$ is a nontrivial sum $\left(B_{1}, T_{1}, \Delta_{1}\right)+$ $\left(B_{2}, T_{2}, \Delta_{2}\right)$, and it is not a sum of 2-twist tangle and a rational tangle. By Lemma 3.3 this implies that $E(T)$ is $\partial$-irreducible. By Lemma 4.1(vi), the annulus $A$ is essential in $E(T)$. Applying Lemma 3.4 to this case, we see that one of the (i), (ii) or (iii) of Lemma 3.4 holds. We separate the discussion into two cases.

CASE 1. (Conclusion (i) of Lemma 3.4 holds, i.e. A can be isotoped to a $\Delta_{1}$-essential annulus in $\left(B_{1}, T_{1}, \Delta_{1}\right)$.) 
Thus $\left(B_{1}, T_{1}, \Delta_{1}\right)$ is $\Delta_{1}$-annular. Let $\partial_{1}, \partial_{2}$ be the two boundary components of the punctured sphere $\partial B \cap E(T)$ which lie on $\partial B_{1}$. By Lemma 4.1 (ii) and (iii), we know that $\alpha=\partial A \cap \partial B$ consists of arcs connecting $\partial_{1}$ and $\partial_{2}$. Since $\Delta$ is disjoint from $\partial A$, this implies that it is disjoint from the connected set $\partial_{1} \cup \partial_{2} \cup \alpha$. It follows that $\Delta$ can be isotoped into $\partial B_{2}$. If $\left(B_{1}, T_{1}, \Delta_{1}\right)$ is in $\mathcal{S}$ then we are done. If $\left(B_{1}, T_{1}, \Delta_{1}\right)$ is not in $\mathcal{S}$, the result follows by induction on the length of the tangle.

CASE 2. ( $A$ can not be isotoped into $E\left(T_{1}\right)$.)

By Lemma 3.4, $A$ is then a union of bigons in $E\left(T_{i}\right)$. Let $A_{i}$ be the annulus $\partial N\left(t_{i}\right) \cap E(T)$, where $t_{i}$ are strings of $T$. Lemma 4.1(i) says that $A$ is disjoint from some $A_{i}$, say $A_{1}$. Look at the nine possible configurations of the bigons in Figure 2.1. By Lemma 2.2 (1) - (3) can not happen. In Case (4) and (5) the bigon intersect the two inner circles in different number of points, so they belong to different $A_{i}$, which is impossible because then $A$ would intersect both $A_{i}$. In Case (6) one can see from the proof of Lemma 2.2 that $\partial A \cap \partial E(T)$ has some circles surrounding $\partial A_{i}$ for some $i$, and has an edge connecting the boundary circles of the other $A_{j}$, so no component of $(\partial E(T) \cap \partial B)-\partial A$ could contain the twice punctured disk $\Delta \cap E(T)$.

Consider Case (9). By the proof of Lemma 2.2 the tangle $T_{1}$ containing this bigon is either a 2-twist tangle, 4 -twist tangle, or a left torus tangle. Since $A$ is essential, $T_{1}$ can not be a 2-twist tangle. In the other cases from Figure 2.4 we see that one of the $A_{i}$, say $A_{1}$ is in $E\left(T_{1}\right)$, and $A$ intersects $A_{1}$. A bigon on the other tangle $T_{2}$ can not be of type (9), otherwise $T$ would have a closed circle component. Hence it is of of type (7) because that is the only other type which has an arc disjoint from the inner circles. But then from the figure we see that $A$ intersects $A_{2}$ as well, contradicting Lemma 4.1. Similarly one can show that Case (8) can not happen.

In Case (7), the tangle is a $( \pm 3)$-twist tangle. There is a unique gluing disk on $\partial B$ which is disjoint from $A$. The curves $A \cap \partial B_{i}$ appear in Figure 2.3. By drawing the picture on $\partial B$, one can see that $T$ is similar to either $R\left[\frac{1}{3},-\frac{1}{3} ; 0\right]$ or $R\left[\frac{2}{3},-\frac{1}{3} ;-\frac{1}{3}\right]$.

Lemma $4.8 A$-wrapping tangle $T$ is algebraic if and only if $k= \pm 1$, in which case $T=R\left[\mp \frac{1}{2}, \pm \frac{1}{3} ; 0\right]$.

Proof. The tangle space $E(T)$ of a wrapping tangle or torus tangle is $\partial$-reducible because of the existence of bigons, so by Lemma 3.3, if $(B, T)$ is homeomorphic to a nontrivial algebraic tangle $\left(B^{\prime}, T^{\prime}\right)$, then $\left(B^{\prime}, T^{\prime}\right)=M\left(\frac{1}{2}, \frac{r}{s}\right)$ for some rational number $r / s$, the exterior of the knotted string of $T^{\prime}$ is the same as the exterior of an $r / s$ 2-bridge knot in $S^{3}$.

Without loss of generality we may assume that the wrapping number of $T$ is $k>0$. The exterior of the knotted arc of $T$ is the exterior of a $(2,2 k+1)$ torus knot in $S^{3}$, which is a 
2-bridge knot with associated rational number $1 /(2 k+1)$. If $T=T^{\prime}$, then $r / s=1 /(2 k+1)$ $\bmod \mathbf{Z}$, i.e. $T$ is the sum of a 2 -twist tangle and a $2 k+1$ twist tangle.

From Figure 1.8 one can see that the induced link $L$ of a wrapping tangle is a trivial knot. Thus there are $\operatorname{arcs} \alpha_{1}, \alpha_{2}$ on $\partial B^{\prime}$ such that $T^{\prime} \cup \alpha_{1} \cup \alpha_{2}$ is a trivial knot in $S^{3}$. On the other hand, $T^{\prime}$ as shown in Figure 4.4(a) has the property that when connecting the ends with vertical lines we get a composite link. According to a theorem of Eudave-Muñoz [5, Theorem 6], if there are $\operatorname{arcs} \alpha_{1}, \alpha_{2}$ on $\partial B^{\prime}$ connecting the ends of $T^{\prime}$ to produce a trivial knot, then $\alpha_{i}$ intersects the circle $C$ in Figure 4.4(a) only once. The knot $K=T^{\prime} \cup \alpha_{1} \cup \alpha_{2}$ can be drawn as in Figure 4.4(b). After changing a single crossing, it becomes a $(2,2 k+1)$ torus knot, which has unknotting number $k$. Hence $K$ can not be a trivial knot unless $k=1$. From Figure 1.8 it is clear that $( \pm 1)$-wrapping tangle is an $R\left[\mp \frac{1}{2}, \pm \frac{1}{3} ; 0\right]$.
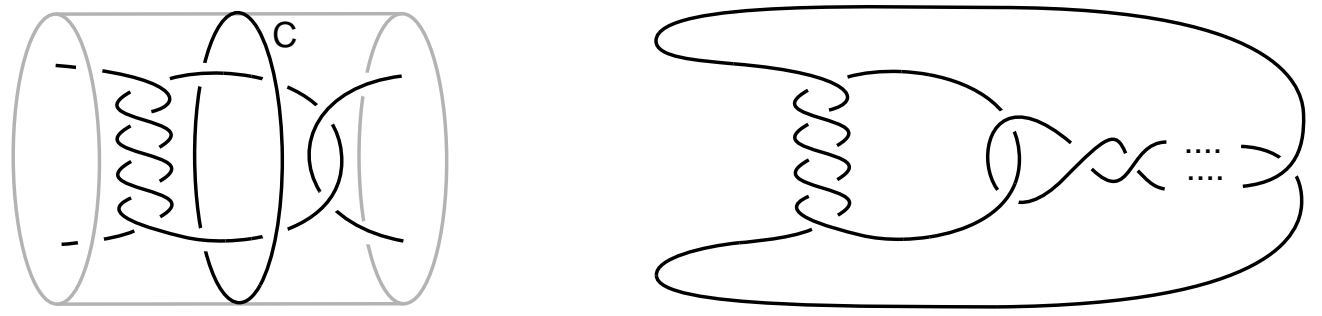

Figure 4.4

Theorem 4.9 A nontrivial algebraic tangle $T$ is nonsimple if and only if one of the following holds.

(a) $T=M\left(\frac{1}{2}, \frac{p}{q}\right)$;

(b) $T=M\left(\frac{1}{q}, \frac{1}{q^{\prime}}\right)$, $q$ and $q^{\prime}$ are odd numbers;

(c) $T=T_{1}+T_{2}$, each $T_{i}$ is $R\left[\frac{1}{2},-\frac{1}{3} ; 0\right]$ or $R\left[-\frac{1}{2}, \frac{1}{3} ; 0\right]$, and the unknotted string of $T_{1}$ is glued to the unknotted string of $T_{2}$;

(d) $T=T_{1}+T_{2}$, and $T_{1} \in \mathcal{S}$.

Proof. Apply Theorem 3.6 to algebraic tangles. Consider the four cases (1) - (4) in the conclusion of Theorem 3.6. Case (1) is the same as (a) above. In case (2), if both $T_{i}$ are odd-twist tangles, we get (b). If one of them is an even-twist tangle, then it is in $\mathcal{S}$, so (d) holds. In case (3), (c) follows from Lemma 4.8. Finally in case (4), $T=T^{\prime}+T^{\prime \prime}$ and $T^{\prime}$ is $\Delta$-annular, so by Proposition $4.7, T^{\prime}$ can be written as $\left(B_{1}, T_{1}, \Delta_{1}\right)+\left(B_{2}, T_{2}, \Delta_{2}\right)$, so that the gluing disk $\Delta$ between $T^{\prime}$ and $T^{\prime \prime}$ is on $\partial B_{2}$, and $T_{1}$ is in $\mathcal{S}$. Therefore, $T$ can be written as a sum $T_{1}+\left(T_{2}+T^{\prime \prime}\right)$ with $T_{1} \in \mathcal{S}$, as described in (d). 


\section{References}

[1] Adams, C. and Reid, A.: Quasi-Fuchsian surfaces in hyperbolic knot complements, preprint.

[2] Bonahon, F. and Siebenmann, L.: Geometric splitting of knots, and Conway's algebraic knots, preprint.

[3] Burde, G. and Ziechang, H.: Knots, de Gruyter Studies in Math. 5, Walter de Gruyter 1985.

[4] Conway, J.: An enumeration of knots and links, and some of their algebraic properties. Computational problems in abstract algebra, pp. 329-358, New York and Oxford: Pergamon 1970.

[5] Eudave-Muñoz, M.: Primeness and sums of tangles, Trans. Amer. Math. Soc. 306 (1988) $773-790$.

[6] Hatcher, A and Thurston, W.: Incompressible surfaces in 2-bridge knot complements, Invent. Math. 79 (1985) 225-246.

[7] Jaco, W.: Lectures on three-manifold topology, Regional conference series in mathematics 43 (1980).

[8] Myers, R.: Simple knots in compact orientable 3-manifolds, Trans. Amer. Math. Soc. 273 (1982) 75-91.

[9] Oertel, U.: Closed incompressible surfaces in complements of star links, Pacific J. Math. 111 (1984) 209-230.

[10] Ruberman, D.: Seifert surfaces of knots in $S^{4}$, Pac. J. Math., 145 (1990) 97-116.

[11] Scharlemann, M.: Producing reducible 3-manifolds by surgery on a knot, Topology 29 (1990) 481-500.

[12] Thurston, W.: The Geometry and Topology of 3-manifolds, lecture notes, 1992 Berkeley edition.

Department of Mathematics, University of Texas at Austin, Austin, TX 78712

\section{CURRENT ADDRESS}

Department of Mathematics, University of lowa, lowa City, IA 52242

E-mail: wu@math.uiowa.edu 